\title{
CAMBios florísticos INDUCIDOS POR LA FRECUENCIA DE FUEGo EN EL Chaco Serrano
}

\author{
LUCAS M. CARBONE ${ }^{1,2^{*}}$, NATALIA AGUIRRE-ACOSTA ${ }^{1}$, JULIA TAVELLA ${ }^{1}$ y RAMIRO \\ AGUILAR ${ }^{1}$
}

\begin{abstract}
Summary: Floristic changes induced by fire frequency in Chaco Serrano. The Sierras Chicas from Córdoba is the mountain range with the highest fire frequency in central Argentina. However, the response to fire frequency of Chaco Serrano plant communities has not yet been assessed. Based on 23-year fire history, we surveyed all vascular plants in unburned, low and high fire frequency sites in the Chaco Serrano district located on Sierras Chicas from Córdoba. We found that fire frequency induces changes in vegetation structure, plant richness, and composition. Specifically, we observed a decrease in total plant richness at sites with a higher number of fire events. All life forms showed decreases in richness to the effects of fire, except the graminoids that showed no changes. In addition, we recorded a change in the species composition between the burned sites (both frequencies) and unburned sites, evidenced through the emergence of indicator species that define both conditions. These results indicate that fire is a strong ecological process that shapes plant communities, selecting species with life history traits (i.e., resprouting and lifespan) that confer adaptative value to new environmental conditions imposed by the regime of high fire frequency.
\end{abstract}

Key words: Fire regime, plant richness, species composition, indicator species, Sierras Chicas.

Resumen: Las Sierras Chicas de Córdoba son el cordón montañoso con mayor frecuencia de fuego del centro de Argentina. Sin embargo, hasta el momento no se ha evaluado cuál es la respuesta de las comunidades de plantas del Chaco Serrano a los efectos de la frecuencia de fuego. En base a 23 años de historia de fuego, evaluamos la fisonomía de la vegetación, la riqueza y la composición de la comunidad de plantas vasculares en sitios no quemados, con baja y alta frecuencia de fuego en el distrito Chaqueño Serrano localizado en las Sierras Chicas de Córdoba. Encontramos que la frecuencia de fuego produce cambios en la estructura de la vegetación, en la riqueza y en la composición florística. Específicamente, observamos una disminución de la riqueza total de especies en sitios con un mayor número de eventos de fuego. Todas las formas de crecimiento mostraron una disminución de su riqueza a los efectos del fuego, a excepción de los graminoides que no mostraron cambios. Además, detectamos un cambio en la composición de especies entre los sitios quemados (ambas frecuencias) y los sitios no quemados, caracterizado por la emergencia de especies indicadoras que definen ambas condiciones. Estos resultados indican que el fuego es un fuerte proceso ecológico que estructura las comunidades vegetales, seleccionando especies con rasgos de historia de vida (i.e., rebrote y ciclo de vida) que confieren valor adaptativo a las nuevas condiciones ambientales que impone el régimen de elevada frecuencia de fuego.

Palabras clave: Régimen de fuego, riqueza de plantas, composición de especies, especies indicadoras, Sierras Chicas.

${ }^{1}$ Instituto Multidisciplinario de Biología Vegetal, CONICET-Universidad Nacional de Córdoba . Av. Velez Sarsfield 299. CC 495, CP 5000. Córdoba, Argentina.

2 Facultad de Ciencias Agropecuarias. Universidad Nacional de Córdoba. Ing. Agr. Felix Aldo Marrone 746. Ciudad Universitaria. CC 509. CP 5000. Córdoba, Argentina. (0351) 4334103.

*autor para correspondencia: Icarbone@agro.unc.edu.ar 


\section{INTRODUCCIÓN}

El fuego es un proceso ecológico y evolutivo presente en la mayoría de los ecosistemas terrestres, el cual modela la distribución, estructura y dinámica de las comunidades vegetales (Bond et al., 2005). La respuesta de la vegetación al fuego depende por un lado de las características de cada incendio, por ejemplo su intensidad y su estacionalidad, así como del régimen de fuego histórico de una región, que incluye además variables temporales como la frecuencia de fuego y el tiempo de retorno (Williams et al., 1999; Govender et al., 2006). Por otro lado, tal respuesta depende de rasgos de historia de vida de las especies vegetales que crecen bajo un determinado régimen de disturbios, tales como el fuego, la herbivoría y la sequía estacional (Milchunas \& Lauenroth, 1993; Davies, 2013). Ante el escenario actual de cambios en el uso de la tierra sumados al cambio climático y al aumento de las igniciones antrópicas, es probable que se produzcan cambios en los regímenes de fuego al afectar su frecuencia y/o intensidad (Pausas \& Keeley, 2009; Keeley et al., 2011; Enright et al., 2015). En efecto, estos cambios pueden afectar la regeneración, el crecimiento y las supervivencia de las especies, modificando drásticamente la estructura de las comunidades vegetales, pudiendo inclusive generar un impacto ecológico fuera del rango de sostenibilidad del sistema (Pausas, 2012; Enright et al., 2015).

La hipótesis del "disturbio intermedio" (Huston, 1979) indica que los niveles intermedios de disturbio promueven la biodiversidad. Aplicada a la ecología del fuego, se predice un pico de diversidad de especies a frecuencias o intensidades de fuego intermedias. Según esta hipótesis, la diversidad disminuye en ausencia de fuego por mayor exclusión competitiva entre las especies y también disminuye en alta frecuencia por mayor mortalidad, de manera que la mayor diversidad es mantenida por frecuencias de fuego intermedias (Huston, 1979). Cuando la frecuencia de fuego es muy elevada, la comunidad estará compuesta por las pocas especies capaces de llegar a la madurez en corto tiempo, y a su vez, la inmigración de especies desde otras partes del paisaje es limitada por el escaso tiempo disponible para el establecimiento exitoso (Connell, 1978). Por el contrario, la ausencia de fuego aumentará la competencia entre especies vegetales, permitiendo que prevalezcan especies dominantes por sobre las especies raras, disminuyendo la riqueza de la comunidad (Connell, 1978). Sin embargo, las especies no necesariamente están adaptadas a los regímenes antrópicos de disturbios como el fuego (Connell, 1978), donde la extinción de especies podría incrementar excesivamente debido a cambios demográficos en respuesta a las condiciones de crecimiento adversas (Enright et al., 2015; Mandai, 2015). Las evidencias empíricas muestran respuestas contradictorias de la diversidad vegetal a los efectos de la frecuencia de fuego dentro de una misma región. Por un lado, se ha observado una alta resiliencia de la comunidad de plantas en pastizales subtropicales ante quemas anuales (Uys et al., 2004). Por otro lado, una disminución de la diversidad vegetal ha sido reportada en pastizales templados (Collins et al., 1995; Li et al., 2013), bosques y sabanas tropicales a altas frecuencias de fuego (Nayak et al., 2014). Si bien existen estudios sobre los efectos del fuego en la vegetación del Chaco Serrano (Verzino et al., 2005; Giorgis et al., 2013; Torres et al., 2014), hasta el momento no se ha evaluado cómo las elevadas frecuencias de fuego actuales afectan la diversidad y composición de la comunidad de plantas en esta ecoregión.

El fuego ha estado presente históricamente en el gran Chaco (Bucher, 1982; Kunst, 2011). Antes de la colonización europea, el paisaje Chaqueño del centro de Argentina estaba constituido por un mosaico de bosques, sabanas y pastizales, mantenidos por fuegos periódicos producidos por los pobladores nativos (Bucher \& Schofield, 1981; Bucher, 1982). Luego de la colonización europea, los antecedentes con respecto a la dinámica del régimen de incendios postulan una disminución de la frecuencia por la introducción de herbívoros domésticos y una consecuente reducción de la biomasa fina (Morello \& Saravia Toledo, 1959; Bucher \& Schofield, 1981; Bucher \& Huszar, 1999). Más recientemente, hacia mediados del siglo pasado, se ha evidenciado un aumento de la frecuencia de fuego asociado a quemas prescritas destinadas al control de leñosas que se extendieron ampliamente en ambientes degradados por la historia de uso (Kunst, 2011). Actualmente, el Chaco es la región fitogeográfica de Argentina con mayor superficie afectada por incendios (PNEF, 2011), y dentro del distrito Chaqueño Serrano, las Sierras Chicas de Córdoba son el cordón serrano 
con mayor frecuencia de fuego (un incendio cada 5-6 años), con un $25 \%$ de su superficie incendiada durante el periodo 1999-2011 (Argañaraz et al., 2015 a; Argañaraz, 2016). La mayor frecuencia de incendios en las Sierras Chicas se debe principalmente a la actividad antrópica, y afecta a matorrales y pastizales debido a sus características de inflamabilidad y a su fisonomía abierta, que favorece la dominancia de especies herbáceas y la propagación del fuego (Argañaraz, 2016).

En comunidades con alta recurrencia de incendios como el Chaco Serrano, la dinámica suelo-vegetación es fuertemente afectada por el régimen de fuego. Se ha reportado para esta región que tanto el tiempo post-fuego como la frecuencia de incendios condicionan las propiedades del suelo (Giorgis et al., 2013; Carbone \& Aguilar, 2016). En este sentido, mientras se ha encontrado que el primero de estos factores no tiene mucha influencia sobre la recuperación de la cobertura arbórea (Giorgis et al., 2013), la frecuencia de fuego puede modelar la divergencia fenotípica de rasgos funcionales a nivel poblacional (Carbone \& Aguilar, 2016) pero aún no ha sido evaluada la respuesta de la comunidad de plantas.

En este sistema montañoso, la principal estrategia de regeneración post-fuego de las plantas leñosas es el rebrote, presentando alta supervivencia y rápida recuperación de su biomasa después del fuego (Torres et al., 2014; Herrero et al., 2016). En efecto, se ha sugerido que comunidades dominadas por especies de plantas rebrotadoras mostrarían poco cambio de la composición de especies después de la ocurrencia de un incendio (Bond \& Midgley, 2001). Sin embargo, especies con capacidad de rebrote pero con formas de crecimiento contrastantes pueden ser favorecidas o perjudicadas por un determinado régimen de fuego, resultando en cambios composicionales a nivel comunitario. Existe evidencia sobre una alta resiliencia de hierbas y pastos a un amplio rango de frecuencias de fuego en sabanas y bosques, mientras que formas de crecimiento leñosas y algunos pastos palatables, con alto valor de conservación y para la producción ganadera, disminuyen su riqueza y abundancia ante fuegos muy frecuentes (Uys et al., 2004; Watson et al., 2009; Nayak et al., 2014). El rol de la frecuencia de fuego al favorecer a formación de comunidades herbáceas y arbustivas más inflamables, propensas a la ocurrencia de futuros incendios (Birk \&
Bridges 1989; Kunst \& Bravo, 2003; Paritsis et al., 2015), cobra central importancia para establecer estrategias de manejo del fuego en los sistemas naturales orientadas a conservar la biodiversidad, los servicios ecosistémicos y al mismo tiempo propiciar el uso sustentable de los recursos naturales (Nayak et al., 2014).

En este trabajo planteamos caracterizar la fisonomía de la vegetación y analizar la composición florística de sitios sujetos a diferente frecuencia de fuego. Para ello medimos la estructura de los estratos de la vegetación, la riqueza total de especies y de diferentes formas de crecimiento, la composición de la comunidad y la ocurrencia de especies indicadoras en escenarios no quemados, de baja frecuencia y de alta frecuencia de fuego en el Chaco Serrano de las Sierras Chicas de Córdoba. De acuerdo a la hipótesis del disturbio intermedio, esperamos encontrar la máxima riqueza de especies en sitios de baja frecuencia de fuego. Por otro lado, planteamos que el incremento en la frecuencia de fuego observado en el Chaco Serrano modifica directamente la estructura de la vegetación e impacta negativamente sobre las condiciones abióticas (Carbone \& Aguilar 2016) lo que genera un fuerte filtro ecológico, donde solo aquellas especies con estrategias de regeneración post-fuego y capaces de alcanzar la madurez rápidamente (i.e., con ciclo de vida corto) conformarán las comunidades sujetas a alta frecuencia de fuego. Por lo tanto, en estas comunidades se observará un predominio de especies herbáceas (hierbas y graminoides) que serán favorecidas por sobre las leñosas (árboles y arbustos). Finalmente, hipotetizamos que la composición de especies vegetales será diferente entre las tres condiciones de frecuencia de fuego.

\section{Materiales y Métodos}

\section{Área de estudio}

El área de estudio se ubica en las laderas orientales de las Sierras Chicas (Córdoba, Argentina), entre las latitudes $31^{\circ} 5^{\prime} 39^{\prime \prime}-31^{\circ} 9^{\prime} 12^{\prime \prime} \mathrm{S}$ y las longitudes $4^{\circ} 20^{\prime} 40^{\prime \prime}$ - $4^{\circ} 24^{\prime} 11^{\prime \prime} \mathrm{O}$, abarcando un área aproximada de $40 \mathrm{~km}^{2}$. Al igual que las demás formaciones montañosas pertenecientes a las Sierras Pampeanas, las Sierras Chicas se caracterizan por presentar orientación nortesur y una elevación desde los 500 a los 1947 
m s.n.m. El clima es de tipo mesotermal, semihúmedo con tendencia a semi-seco de montaña, con temperaturas mínimas y máximas promedios entre 10 y $25{ }^{\circ} \mathrm{C}$ (Capitanelli, 1979). La precipitación promedio ronda los $900 \mathrm{~mm}$ anuales, existiendo una considerable variabilidad entre años (600-1500 mm, Flia. Malanca, com. pers.; Giorgis et al., 2013). Las lluvias se concentran en la estación cálida, entre los meses de octubre y marzo, delimitando un claro período de déficit hídrico durante otoño e invierno, pudiendo extenderse hasta el inicio de primavera.

El Chaqueño Serrano (Cabrera, 1971) se caracteriza por presentar una vegetación de tipo bosque de montaña subtropical seco, que se entremezcla con matorrales y pastizales, conformando una estructura compleja de fisonomías (Luti et al., 1979; Bucher, 1982; Gavier \& Bucher, 2004). El área de estudio se ubica en el primer piso altitudinal de vegetación (500-1300 m. s.n.n), denominado Bosque Serrano (Luti et al., 1979). Las especies vegetales que dominan el estrato arbóreo son Schinopsis lorentzii y Lithraea molleoides, acompañadas principalmente por Acacia caven, Celtis ehrenbergiana, Condalia spp., Ruprechtia apetala y Zanthoxylum coco (Cabrera, 1971). Entre los arbustos se encuentran Baccharis spp., Caesalpinia gilliesii, Colletia spinosissima y Schinus fasciculatus; y entre las herbáceas abundan dicotiledóneas junto a graminoides de los géneros Festuca, Nassella y Jarava. Debido a las presiones de incendios en combinación con pastoreo, agricultura y urbanización (Gavier \& Bucher, 2004), esta zona se ha transformado en una franja altitudinal de transición entre el bosque nativo propiamente dicho (característico de las zonas más bajas) y los pastizales (típicos de las zonas más elevadas), entremezclados con fisonomías arbustivas como el romerillal, matorrales abiertos y cerrados (sensu Giorgis, 2011). Sumado a esto, uno de los principales factores que contribuye a la reducción de la extensión original de la vegetación nativa es la invasión de especies leñosas exóticas como Cotoneaster spp., Pyracantha spp. y especialmente Ligustrum lucidum, que forma bosques monotípicos (Giorgis, 2011; Giorgis \& Tecco, 2014).

En esta región, los incendios forestales típicamente ocurren al finalizar la estación seca, propiciados por el aumento de las temperaturas, la escasa humedad de la biomasa combustible y vientos que alcanzan altas velocidades (Bravo et al., 2001). Existen dos factores importantes que predisponen a un incremento en la actividad del fuego en esta región, modificando su régimen (Kunst \& Bravo, 2003). Por un lado el aumento en las precipitaciones en las últimas décadas ha provocado un aumento en la producción de biomasa combustible que favorece el desarrollo de los incendios (Miglietta, 1994; Bravo et al., 2010). Por otro lado, si bien Córdoba es una de las provincias con mayor ocurrencia de tormentas con descargas eléctricas del país (Nicora et al., 2013; Garreaud et al., 2014), las principales causas de la extensa superficie afectada cada año son el aumento de las igniciones antrópicas, accidentales o intencionales, asociados a la expansión poblacional sobre las áreas naturales. Asimismo, la introducción de gramíneas exóticas, como Eragrostis curvula (Fuentes et al., 2013) y Megathyrsus maximus (Carbone. obs. pers.), puede traer aparejado profundos cambios en el régimen de fuego a través del gran potencial de producción de materia seca, características de tolerancia al fuego, alta inflamabilidad y desplazamiento de la vegetación nativa (Kunst, 2011; Jaureguiberry, 2012). Si bien no existen datos empíricos de la historia de incendios para una ventana temporal amplia que permita conocer los regímenes de fuego anteriores a la presencia del hombre, las frecuencias de fuego reportadas en la actualidad son consideradas entre las más elevadas de Argentina central (Argañaraz, 2016).

\section{Selección de sitios de muestreo}

Se hizo un gran esfuerzo de búsqueda para seleccionar sitios con diferente frecuencia de fuego pero que tuvieran el mismo tiempo trascurrido desde el último incendio. Para lograr esto, se compiló la historia de fuego de los últimos 23 años del área de estudio con centro en el Dpto. Colón de la provincia de Córdoba, a partir de diferentes fuentes de información. Se utilizaron registros de fuegos realizados por M.A. Giorgis en colaboración con Defensa Civil de Río Ceballos para el período 1991-2011 (Giorgis et al., 2013). Luego se procedió a la corroboración de la precisión de límites geográficos de los eventos de fuego a partir de vectores de incendios basados en imágenes satelitales Lansat TN y ETM, con una resolución espacial de 30 m, para el periodo 1999 - 


\section{M. Carbone et al. - Cambios florísticos inducidos por el fuego}

2011 (Argañaraz et al., 2015 b). Finalmente, se combinaron entrevistas con residentes locales de la zona rural y observaciones in situ para corroborar definitivamente los registros de incendios hasta el año 2014. En base a esto, se seleccionaron nueve sitios que fueron clasificados en tres escenarios de fuego: (i) tres sitios no quemados, (ii) tres sitios de baja frecuencia de fuego, con 1 a 2 eventos de fuego; y (iii) tres sitios de alta frecuencia de fuego, con 3 a 4 incendios durante el periodo de 23 años analizado. Es importante destacar que el factor de análisis de este trabajo (frecuencia de fuego) está conformado por tres escenarios de fuego, los que se hallan representados por tres réplicas espaciales del mismo régimen de fuego, pero con eventos de fuego diferentes entre sitios. Además, el tiempo transcurrido desde el último evento de fuego es similar para la mayoría de los sitios quemados (3 años). Debido a que no se han observado marcas de fuego sobre los árboles maduros de los sitios no quemados, se asume que estos sitios no se incendian desde hace 40-50 años. Sin embargo, de acuerdo a su fisonomía relativamente abierta, no se podría descartar completamente la ocurrencia de antiguos incendios. Los detalles de la historia de fuego de los sitios, su ubicación geográfica y características topográficas pueden consultarse en Carbone \& Aguilar (2016). Si bien no tenemos indicio sobre cómo fue la composición florística original de los sitios, la selección de estos fue realizada en base a los siguientes criterios tendientes a minimizar las diferencias atribuidas a la identidad del sitio de muestreo: relativa cercanía (distancia promedio entre sitios de $1530 \mathrm{~m}$ ), altitud (rango de elevación entre 824-1206 m s.n.m.), posición topográfica (ladera media), exposición ( $\mathrm{N}$, en su mayoría) y pendiente. Todos los sitios seleccionados pertenecen a propiedades privadas y tres de ellos están incluidos en la Reserva Hídrica Natural Parque La Quebrada. Debido a que la cría de ganado bovino es la principal actividad productiva de la región, se estimó la carga ganadera mediante el conteo de deposiciones de vacunos y equinos en un área de $500 \mathrm{~m}^{2}$ en cada uno de los sitios. A los fines de identificar algún efecto sinérgico con la frecuencia de fuego en la respuesta de la vegetación, la carga ganadera de los sitios fue incluida en el análisis de composición de especies (ver Análisis de datos).

\section{Censos de vegetación}

Con el objetivo de caracterizar la comunidad vegetal en los diferentes escenarios de frecuencia de fuego, se realizó un censo de vegetación durante febrero de 2014 en una parcela de 500 $\mathrm{m}^{2}$ en cada uno de los nueve sitios de estudio. El tamaño de la parcela fue definido en base a estudios fitosociológicos previos que determinan el área mínima necesaria para incluir casi la totalidad de las especies en el Chaco Serrano (Cabido et al., 1991). En cada parcela se registró la altura y cobertura promedio de los diferentes estratos de vegetación (arbóreo, arbustivo y herbáceo) y la presencia de todas las especies de plantas vasculares. Las especies registradas fueron identificadas siguiendo diferente bibliografía taxonómica de la especialidad de cada grupo. La actualización de los nombres científicos se realizó según la versión on-line del Catálogo de las Plantas Vasculares del Cono Sur (Zuloaga et al. 2008), al igual que la categorización de cada especie según su origen (nativas, endémicas, exóticas y cosmopolitas) y su forma de crecimiento: árboles, arbustos, enredaderas, hierbas y graminoides (incluyendo Poaceae y Cyperaceae). Los ejemplares de referencia de las especies vegetales censadas fueron depositados en el Herbario de la Facultad de Ciencias Agropecuarias, de la Universidad Nacional de Córdoba (ACOR).

\section{Análisis de datos}

Para corroborar la confiabilidad del muestreo en base al tamaño muestral indicado por Cabido et al. (1991) se calcularon estimadores no paramétricos de riqueza de especies basados en la ocurrencia de las mismas en cada escenario de fuego (ICE, Chao2, Jacknife 1 y 2), utilizando el programa EstimateS (Colwell, 2013). El porcentaje de confiabilidad del muestreo se calculó como la proporción entre estos valores estimados de riqueza y el número observado de especies en cada condición. Para comparar la riqueza de especies entre los tres escenarios de fuego se realizó una estimación de diversidad no asintótica a través de curvas de rarefacción y extrapolación, estimando el número de especies en base a una similar completitud del muestreo en lugar de un tamaño similar (Chao \& Jost, 2012). Este método permite hacer comparaciones robustas de la riqueza de especies entre muestras incompletas. Se estimó la riqueza para cada condición a un misma completitud con un remuestreo de 100 bootstraps 
y se estimó el intervalo de confianza del 95\%. Este análisis fue realizado usando el programa iNEXT Online (Chao \& Jost, 2012, Chao et al., 2016).

Para analizar si la riqueza de especies de cada forma de crecimiento (árboles, arbustos, enredaderas, hierbas y graminoides) difirió entre escenarios de fuego, se utilizaron modelos lineales generalizados (función $\mathrm{glm}$ del paquete stats) con distribución de errores Poisson en entorno R ( R Core Team, 2016). La significancia del factor fijo (frecuencia de fuego) sobre el número de especies fue probada mediante comparación del modelo global con un modelo nulo anidado mediante pruebas de razón de verosimilitud (likelihood ratio test, LRT). Las diferencias entre los niveles del factor fijo fueron evaluadas con el estadístico Z (Wald). Previo a la ejecución de estos modelos se comprobaron los supuestos de normalidad de residuos y homogeneidad de varianzas para la correcta interpretación de los resultados.

Por otra parte, para comparar la similitud en la composición de especies entre los escenarios de fuego, se construyó una matriz con valores de disimilitud de Jaccard a partir de datos de presencia de las especies en cada sitio. En base a esto, se realizó un análisis multivariado de la varianza con permutaciones (PERMANOVA), utilizando 999 permutaciones, para determinar si la composición de especies fue diferente entre escenarios de fuegos. Este análisis permite particionar los valores de disimilitud entre fuentes de variación y ajustar un modelo lineal con diferentes variables explicativas. Además del factor fuego, también comprobamos si la composición de especies fue afectada por la carga ganadera (número de deposiciones) y por la altitud de los sitios. Se realizaron comparaciones entre pares de escenarios de fuego. Asimismo, para analizar las diferencias en composición de modo gráfico, se realizó un análisis de ordenamiento NMDS (Non Metric Multidimensional Scaling) utilizando las medidas de disimilitud calculadas. Todos los análisis de composición de especies fueron realizados utilizando el paquete vegan en entorno R (Oksanen et al., 2016). Por último, se realizó un análisis de especies indicadoras mediante el cálculo del valor de indicación (IndVal) a partir de datos de ocurrencia con el paquete indicspecies (De Cáceres \& Legendre, 2009) en R. Este método combina las medidas de especificidad de una especie a un determinado hábitat y su fidelidad dentro de esa condición. Los valores de indicación oscilan entre 0 (ninguna indicación) y 100 (indicación perfecta). La indicación perfecta significa que la presencia de una especie coincide sin error con una condición del hábitat en particular. En general, una especie se considera buena indicadora cuando los valores de IndVal son mayores a $70 \%$, mientras que valores entre 40 y $70 \%$ suelen considerarse especies detectoras, es decir especies que cambian su frecuencia ante la presencia de una modificación ambiental pero sin desaparecer como lo haría una buena especie indicadora.

\section{Resultados}

Los sitios no quemados estuvieron representados por una fisonomía de vegetación intermedia entre un matorral cerrado y un bosque nativo cerrado, caracterizados por una cobertura promedio del estrato arbóreo, arbustivo y herbáceo del 55, 39 y $92 \%$, respectivamente, y una altura promedio del estrato arbóreo de 4,2 m (Tabla 1). En contraste, los sitios quemados tanto de baja como de alta frecuencia de fuego estuvieron conformados por una fisonomía de vegetación en matorral, con una escasa cobertura del estrato arbóreo $(1,3 \%)$, una variable cobertura del estrato arbustivo que promedió el $19 \%$ y una cobertura herbácea del $76 \%$. La altura promedio del estrato más representativo (arbustivo) fue de 1,1 m (Tabla 1). En síntesis, la vegetación de ambos escenarios quemados fue más abierta, baja y con mayor dominancia relativa del estrato arbustivo y herbáceo por sobre el arbóreo.

Se registró un total de 268 taxones (incluyendo especies, subespecies, variedades y formas) en los nueve sitios de muestreo, agrupados en 63 familias de plantas vasculares (Apéndice 1). De ellos, 197 taxones fueron nativos, 44 endémicos, 25 exóticos y 2 cosmopolitas. En cuanto a la forma de crecimiento, se observaron 18 especies de árboles, 24 especies de enredaderas, 47 de arbustos, 135 de hierbas y 44 especies de graminoides (agrupando Poaceae y Cyperaceae).

En el escenario no quemado se registraron 107 especies de plantas para el promedio de los tres sitios, seguido por el escenario de baja frecuencia de fuego con 88 especies y finalmente el escenario de alta frecuencia de fuego, con 64 especies registradas (Tabla 2). De acuerdo a 
Tabla 1. Eventos de fuego ocurridos durante el periodo 1991-2014, cobertura y altura promedio de los estratos de vegetación (arbóreo, arbustivo y herbáceo) y carga ganadera (número de deposiciones de ganado vacuno y equino) en diferentes escenarios de frecuencia de fuego del Chaco Serrano.

\begin{tabular}{|c|c|c|c|c|c|c|c|c|}
\hline \multirow{2}{*}{$\begin{array}{l}\text { Escenario } \\
\text { de fuego }\end{array}$} & \multirow{2}{*}{$\begin{array}{l}\text { Eventos } \\
\text { de fuego }\end{array}$} & \multicolumn{3}{|c|}{ Cobertura estrato (\%): } & \multicolumn{3}{|c|}{ Altura estrato $(m)$ : } & \multirow{2}{*}{$\begin{array}{c}\text { Carga } \\
\text { ganadera }\end{array}$} \\
\hline & & arbóreo & arbustivo & herbáceo & arbóreo & arbustivo & herbáceo & \\
\hline \multicolumn{9}{|c|}{ No quemado } \\
\hline Sitio 1 & 0 & 38 & 63 & 100 & 5 & 1.8 & 0.6 & 4 \\
\hline Sitio 2 & 0 & 38 & 16 & 88 & 4 & 1.3 & 0.5 & 11 \\
\hline Sitio 3 & 0 & 88 & 38 & 88 & 3.5 & 1.5 & 0.6 & 5 \\
\hline \multicolumn{9}{|c|}{ Baja frecuencia de fuego } \\
\hline Sitio 4 & 1 & 3.5 & 16 & 38 & 1.5 & 1 & 0.5 & 8 \\
\hline Sitio 5 & 2 & 0 & 3.5 & 88 & 0 & 1 & 0.5 & 16 \\
\hline Sitio 6 & 2 & 3.5 & 16 & 88 & 5 & 1.5 & 0.7 & 4 \\
\hline \multicolumn{9}{|c|}{ Alta frecuencia de fuego } \\
\hline Sitio 7 & 3 & 0.5 & 63 & 88 & 1.5 & 1.3 & 0.6 & 12 \\
\hline Sitio 8 & 4 & 0 & 5 & 63 & 0 & 0.8 & 0.4 & 27 \\
\hline Sitio 9 & 4 & 0 & 10 & 88 & 0 & 1 & 0.7 & 0 \\
\hline
\end{tabular}

los diferentes estimadores de confiabilidad del muestreo (Apéndice 2), el número total de especies observadas en el escenario no quemado representó entre el $55 \%$ y $73 \%$ de las especies esperadas, en el escenario de baja frecuencia de fuego entre $69 \%$ y $85 \%$, y en la condición de alta frecuencia de fuego entre $50 \%$ y $72 \%$. Si bien estos valores indican una confiabilidad de muestreo intermedia a alta, los diferentes rangos de confiabilidad sugieren que para un mismo esfuerzo de muestreo $\left(500 \mathrm{~m}^{2}\right)$ la completitud del muestreo fue levemente diferente en los distintos escenarios de fuego. La riqueza de especies estimada para una completitud de muestreo equiparable del $\sim 75 \%$ (interpolación para baja frecuencia de fuego) fue significativamente menor en los sitios de baja y alta frecuencia de fuego con respecto al escenario no quemado (Tabla 2). Esto se puede observar a partir de la no superposición de los intervalos de confianza entre las curvas de rarefacción (Fig. 1). Por el contrario, no se

Tabla 2. Riqueza de especies observada, riqueza estimada por extrapolación e interpolación, e intervalos de confianza (IC) al 95\% para una completitud de muestreo del $75 \%$ en diferentes escenarios de frecuencia de fuego del Chaco Serrano. Letras diferentes indican diferencias significativas de la riqueza de especies estimada entre escenarios de fuego de acuerdo a la no superposición de los IC.

\begin{tabular}{|lccccc|}
\hline \multicolumn{1}{|c}{ Escenario de fuego } & $\begin{array}{c}\text { Riqueza } \\
\text { observada }\end{array}$ & $\begin{array}{c}\text { Riqueza } \\
\text { estimada }\end{array}$ & IC inferior & IC superior \\
\hline No quemado & 107 & $158^{\mathrm{a}}$ & 145.57 & 170.43 \\
Baja frecuencia de fuego & 88 & $108^{\mathrm{b}}$ & 100.63 & 116.04 \\
\hline Alta frecuencia de fuego & 64 & $114^{\mathrm{b}}$ & 104.52 & 123.48 \\
\hline
\end{tabular}




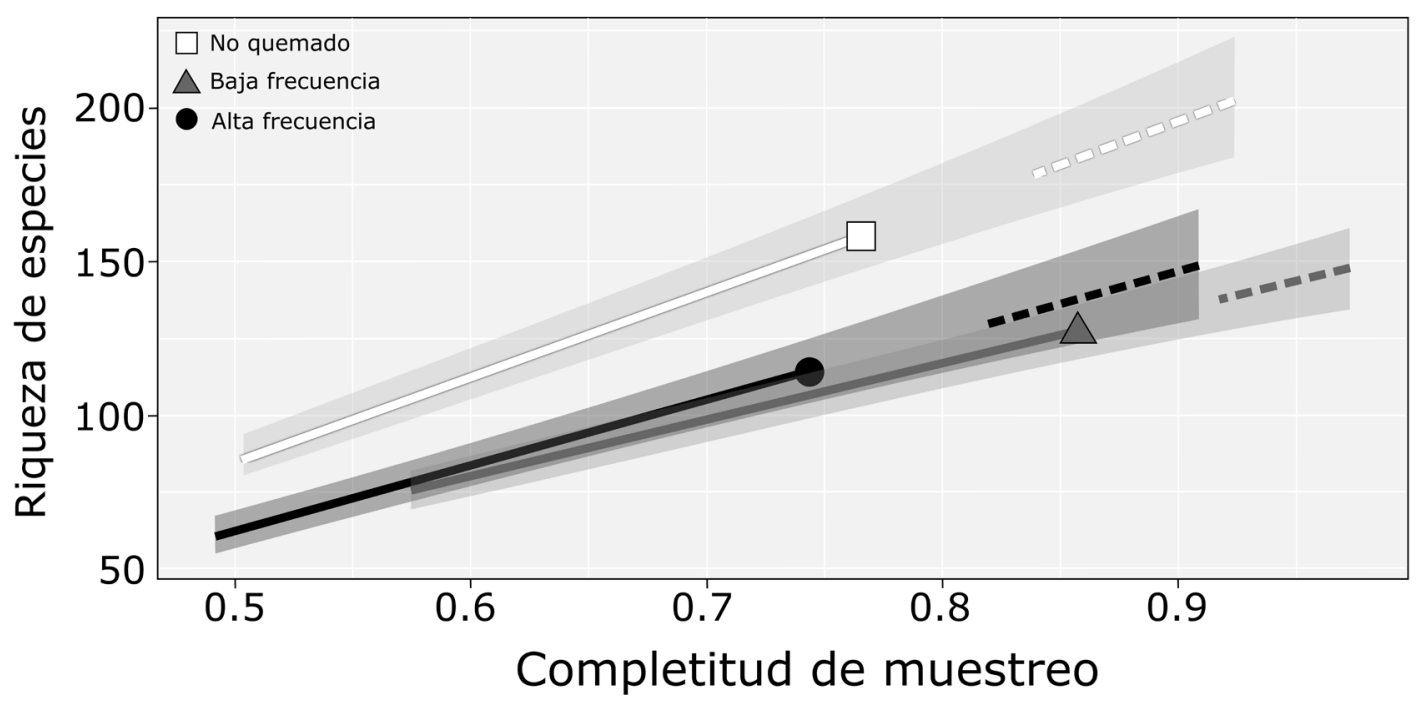

Fig. 1. Riqueza de especies estimada para una muestra rarefaccionada y extrapolada en función de la completitud del muestreo para escenarios no quemados, de baja y de alta frecuencia de fuego. Las líneas continuas corresponden a interpolación de riqueza de especies, las punteadas a extrapolación y las áreas sombreadas a intervalos de confianza del 95\% (basados en bootstrap con 100 remuestreos).

encontraron diferencias en la riqueza de especies entre ambos escenarios quemados (Fig. 1). Por otro lado, el fuego afectó significativamente la riqueza de todas las formas de crecimiento $\left(\chi^{2}>10.128\right.$, $p<0.006)$ excepto la de graminoides $\left(\chi^{2}>2.080\right.$, $p=0.353)$. El número de especies arbóreas, como así también su cobertura, mostraron una drástica reducción $(>50 \%)$ en el escenario de alta frecuencia de fuego en relación a la condición no quemada (Fig. 2). Respecto a la riqueza de arbustos y enredaderas, ambos escenarios quemados mostraron menor número de especies que los sitios no quemados (Fig. 2). La riqueza de especies herbáceas sólo disminuyó en sitios de alta frecuencia de fuego, siendo similar entre los escenarios de baja frecuencia y no quemado. Finalmente, el número de especies graminoides fue similar entre todos los escenarios de fuego (Fig. 2).

Según el análisis estadístico de similitud, la composición florística en términos de ocurrencia de especies fue significativamente explicada por la frecuencia de fuego $\left(R^{2}=0.342, p=0.017\right)$, mientras que las variaciones en composición no estuvieron relacionadas a la carga ganadera de los sitios $\left(R^{2}=0.136, p=0.121\right)$. La composición de especies resultó diferente entre el escenario no quemado y los escenarios de baja frecuencia de fuego $\left(R^{2}=0.335\right.$, $p=0.001)$ y de alta frecuencia de fuego $\left(R^{2}=0.263\right.$, $p=0.001)$, mientras que la composición entre ambas condiciones quemadas fue similar $\left(R^{2}=0.237\right.$, $p=0.201)$. El escenario no quemado presentó 79 especies exclusivas, mientras que en los escenarios de baja y alta frecuencia de fuego se registraron considerablemente menos especies exclusivas (Fig. 3). Los sitios no quemados compartieron 103 especies con los sitios sujetos a baja frecuencia de fuego y 90 con los sitios más frecuentemente quemados, al mismo tiempo que ambos escenarios quemados presentaron 86 especies comunes. Finalmente, el 25 $\%$ del total de las especies (67 especies) estuvieron presentes en todos los escenarios (Fig. 3). Las diferentes composiciones de los ensambles de plantas también pueden corroborarse visualmente a partir de la separación espacial de las comunidades sujetas a diferentes frecuencias de fuego en el gráfico de ordenamiento NMDS (Fig. 4).

El análisis de especies indicadoras mostró que 5 de los 268 taxones resultaron especies indicadoras del escenario no quemado (IndVal $>70 \%, p<0.05$ ) mientras que no se observaron especies indicadoras de los escenarios de baja y de alta frecuencia de fuego (Tabla 3a). Sin embargo, debido a que la composición de especies fue similar entre escenarios de baja y alta frecuencia de fuego, se decidió considerar estas dos condiciones de frecuencia 


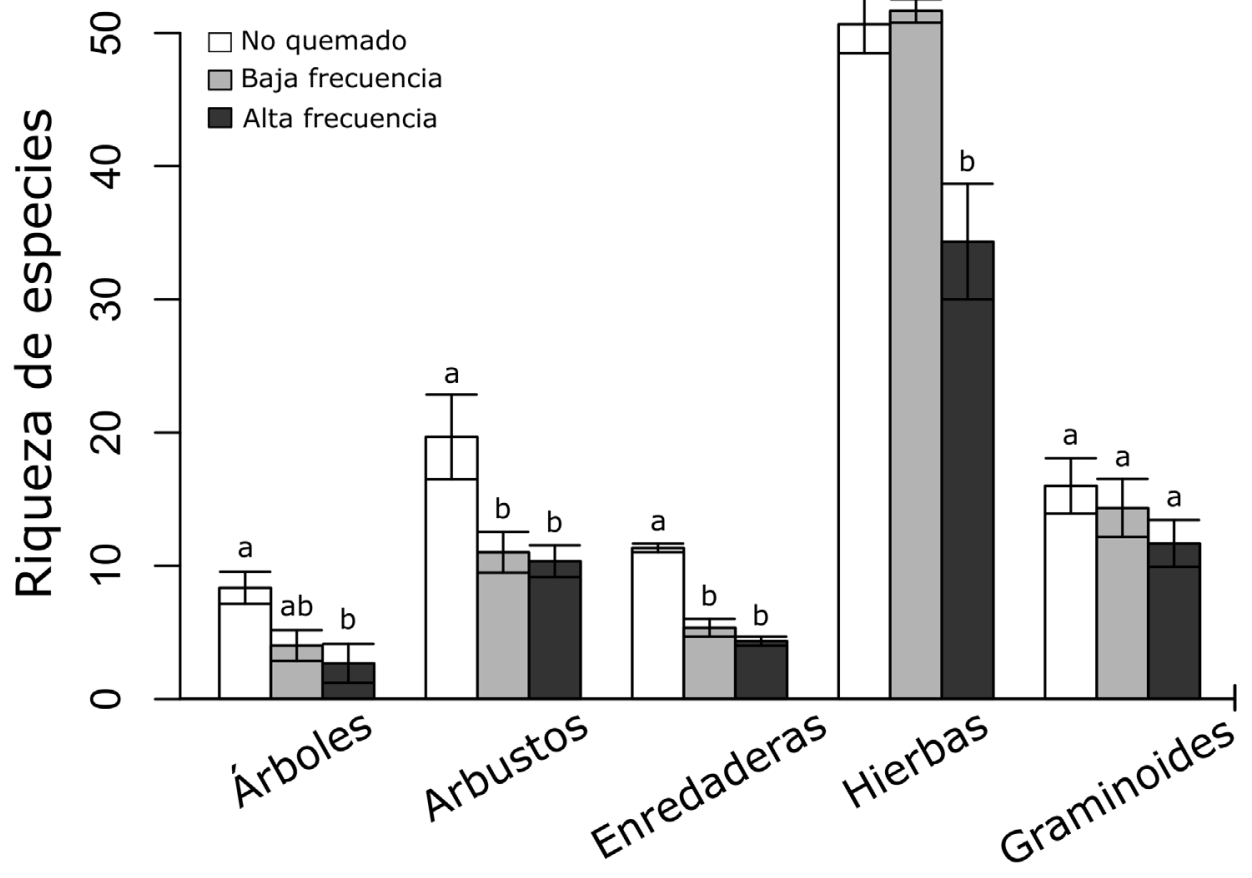

Fig. 2. Riqueza de especies de diferente forma de crecimiento en escenarios no quemados, de baja y de alta frecuencia de fuego. Letras distintas en una misma forma de crecimiento indican diferencias significativas $(p<0.05)$ entre escenarios de fuego de acuerdo a modelos lineales generalizados.

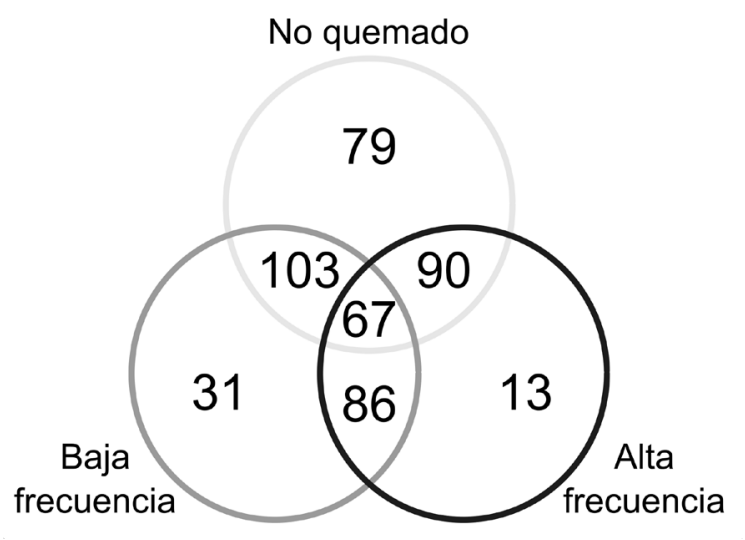

Fig. 3. Diagrama de Venn del número de especies exclusivas y compartidas entre escenarios no quemados, de baja y de alta frecuencia de fuego. Los números indican la cantidad de especies exclusivas de cada escenario de fuego, comunes a dos escenarios de fuego y comunes a las tres condiciones sobre un total de 268 especies registradas. de fuego como una sola condición quemada y compararla con el escenario no quemado. En base a este análisis, el número de especies indicadoras de la condición no quemada se incrementó a 10 especies y además se observó la emergencia de una especie indicadora de la condición quemada (Tabla 3b).

Finalmente, es importante destacar que dos de los taxones registrados, Solanum viarum Dunal (Solanaceae) y Pleopeltis tweediana (Hook.) A.R. Sm. (Polypodiaceae), corresponden a nuevos registros para la flora nativa de Córdoba, los que solamente estuvieron presentes en sitios no quemados (Apéndice 1).

\section{Discusión}

Evaluar la respuesta de la vegetación a factores claves como la frecuencia de fuego es de central importancia para predecir la dinámica de las comunidades vegetales, establecer prioridades de 


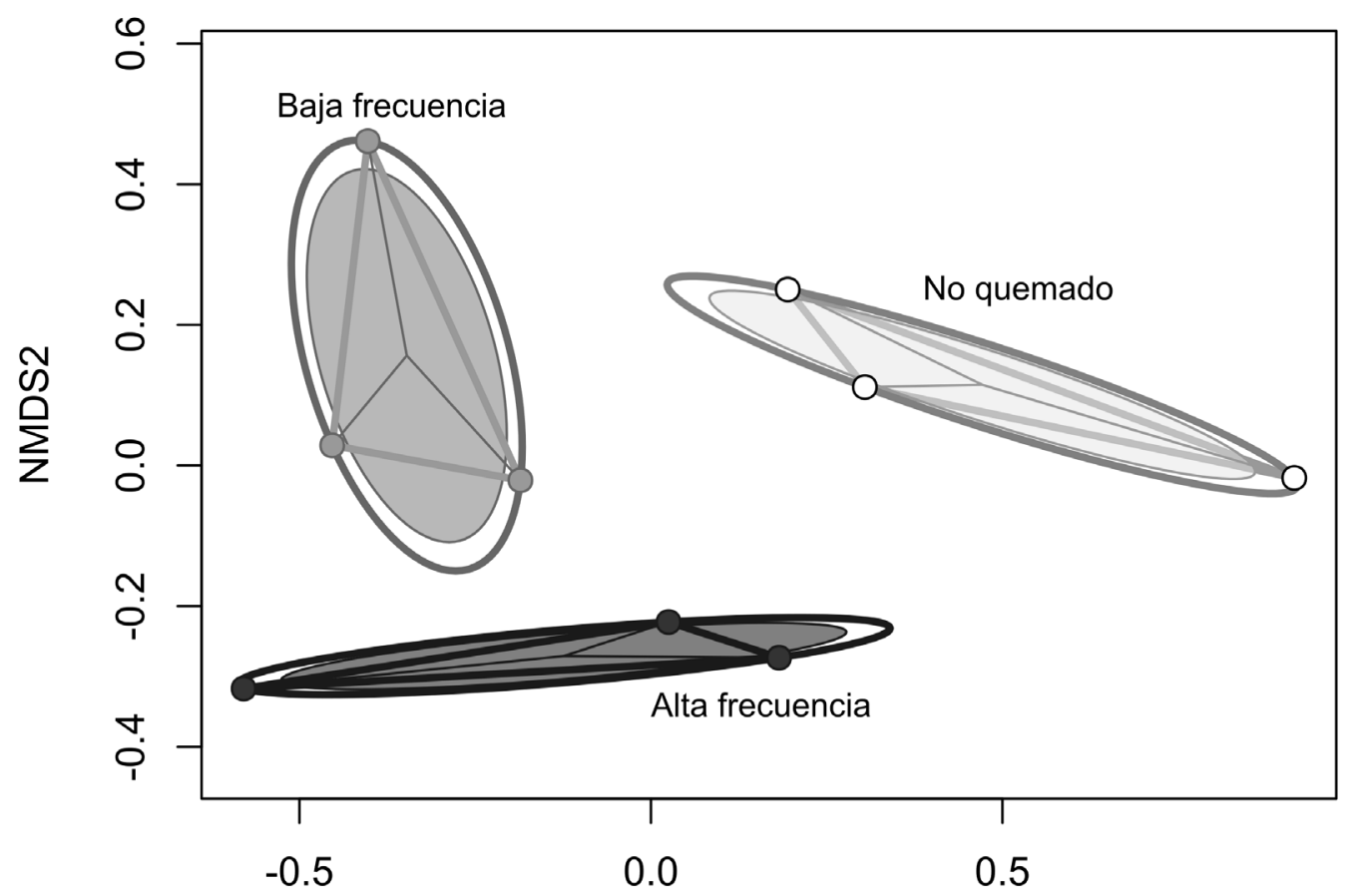

NMDS1

Fig. 4. Gráfico de ordenamiento NMDS representando a los sitios (puntos) de diferentes escenarios de fuego: no quemado, baja frecuencia de fuego y alta frecuencia de fuego. La separación de elipses que agrupan sitios sujetos al mismo régimen de fuego indica diferencias en la composición de especies. El gráfico fue construido con medidas de disimilitud de Jaccard.

conservación y estrategias de manejo sustentable en el contexto de los cambios antrópicos en los regímenes de fuego. Este estudio evidencia que la frecuencia de fuego induce cambios en la estructura de la vegetación y en la riqueza y composición florística en el Chaco Serrano, con una disminución de la riqueza de especies y un cambio en la composición de especies en sitios quemados (ambas frecuencias) versus los sitios no quemados. El presente trabajo constituye el primer reporte florístico que demuestra el rol del fuego como un fuerte proceso estructurador de las comunidades de plantas en el Chaco Serrano.

A escala global la diversidad vegetal responde positivamente con la actividad del fuego, debido a que este retrasa la exclusión competitiva, y genera heterogeneidad de ambientes y nuevos nichos (Pausas \& Ribeiro, 2017). Sin embargo, la respuesta al fuego a escala local puede variar entre biomas y ecosistemas (Pausas \& Ribeiro, 2017). Nuestro trabajo muestra que en base a 23 años de registro de la frecuencia de fuego, la riqueza vegetal no responde de acuerdo con lo predicho por la hipótesis de disturbio intermedio (Connell, 1978; Huston, 1979), pues aquí observamos la menor riqueza de especies tanto en los sitios de baja como de alta frecuencia de fuego. Este patrón de respuesta concuerda con estudios previos desarrollados en ecosistemas boscosos y pastizales de África (Uys et al., 2004) y Australia (Lunt \& Morgan, 2002) sujetos a incrementos en la frecuencia de fuego. Los trabajos que evaluaron los efectos del fuego sobre la diversidad de plantas en el Chaco Serrano analizaron exclusivamente al factor tiempo post-fuego (Verzino et al., 2005; Giorgis et al., 2013). Estos estudios reportan un aumento de la diversidad de especies en los sitios quemados con respecto a sitios no quemados y cambios en 
Tabla 3. Especies indicadoras de acuerdo al análisis de (a) tres condiciones de frecuencia de fuego: no quemado, baja y alta frecuencia de fuego, y (b) de dos condiciones contrastantes: no quemado y quemado. IndVal: valor de indicación calculado con datos de ocurrencia de las especies. Valores de IndVal=100 sugieren indicación perfecta, e IndVal>70 sugieren buena indicación de una especie para una condición.

\begin{tabular}{|ccccc|}
\hline Especie & Abundancia máxima & Condición & IndVal & Valor $\mathbf{p}$ \\
\hline a & 100 & No quemado & 100 & 0.037 \\
Araujia odorata & 100 & No quemado & 100 & 0.037 \\
Chromolaena arnottiana & 100 & No quemado & 100 & 0.037 \\
Dicliptera squarrosa & 100 & No quemado & 100 & 0.037 \\
Euphorbia acerensis & 100 & No quemado & 100 & 0.037 \\
\hline Lorentzianthus viscidus & 100 & Quemado & 83.3 & 0.04 \\
Abutilon pauciflorum & 100 & No quemado & 100 & 0.011 \\
Araujia odorata & 86 & No quemado & 85.7 & 0.05 \\
Celtis ehrenbergiana & 100 & No quemado & 100 & 0.011 \\
Chromolaena arnottiana & 100 & No quemado & 100 & 0.011 \\
Dicliptera squarrosa & 100 & No quemado & 100 & 0.011 \\
Euphorbia acerensis & 86 & No quemado & 85.7 & 0.055 \\
Heterosperma ovatifolium & 86 & No quemado & 85.7 & 0.05 \\
Janusia guaranitica & 100 & No quemado & 100 & 0.011 \\
Lorentzianthus viscidus & 86 & No quemado & 85.7 & 0.05 \\
Salpichroa origanifolia & 86 & No quemado & 85.7 & 0.04 \\
\hline Tillandsia capillaris & & & &
\end{tabular}

la composición vegetal que no son explicados por el tiempo post-fuego (Giorgis et al., 2013). Tal patrón de incremento de la diversidad en respuesta al fuego puede deberse a que los ambientes no quemados seleccionados como comunidad clímax en esos estudios corresponden a fisonomías de bosque serrano en estadios sucesionales tardíos (bosques nativos maduros; ver Giorgis, 2011), los cuales en general presentan una baja diversidad de especies. Si bien en nuestro estudio no evaluamos este tipo de fisonomías boscosas en donde es muy poco probable la ocurrencia de incendios, el fuego ejerce un potente efecto estructurador entre matorrales y bosques nativos sujetos a diferente frecuencia de fuego.

A pesar de la tendencia general a una disminución del número de especies con el aumento en la frecuencia de incendios, las distintas formas de crecimiento respondieron diferencialmente a este disturbio, en coincidencia con la hipótesis originalmente planteada en torno a las formas de vida. Los árboles, arbustos, enredaderas y hierbas mostraron una menor riqueza en sitios con alta frecuencia de incendios, mientras que la ocurrencia de especies graminoides (i.e., Poaceae y Cyperaceae) mostró una gran estabilidad a través de los regímenes de fuego. Una respuesta diferencial de las formas de crecimiento a los efectos del fuego ha sido reportada previamente para el Chaco Serrano de las laderas occidental de las Sierras Chicas (Verzino et al., 2005). El aumento de la riqueza de herbáceas junto con la reducción de especies leñosas en respuesta al fuego ha sido extensamente reportado para muchos ecosistemas del mundo, postulando al fuego como un factor ecológico clave para la conservación de la biodiversidad en pastizales, sabanas y matorrales (e.g., Kunst et al., 2003; Veldman et al., 2013). 
Nuestros resultados son parcialmente congruentes con esto, debido a que ninguna forma de crecimiento mostró un aumento de su riqueza. Sin embargo, las especies graminoides fueron resilientes a los efectos de diferentes frecuencias de fuego y las especies herbáceas solo ante una frecuencia baja. Tal respuesta puede deberse a que la mayoría de estas especies poseen un ciclo de vida corto en comparación con las otras formas de crecimiento. Por lo tanto, las altas frecuencia de fuego no solo favorecerían a especies tolerantes al fuego (i.e., rebrotadoras) sino también a aquellas especies que lo evitan, dado que pueden establecerse rápidamente en el post-fuego temprano desde sitios vecinos no quemados y reproducirse en el intervalo entre fuegos. En consecuencia, el régimen de alta frecuencia de fuego en las Sierras Chicas promueve la generación de comunidades mayormente herbáceas (pastizales) o arbustivas (matorrales) debido a que rasgos de historia de vida podrían conferir valor adaptativo a las especies en zonas con incendios recurrentes. Este tipo de vegetación posee una mayor continuidad de la biomasa y genera condiciones microclimáticas (mayor insolación y exposición al viento, menor humedad) más conductivas para el fuego que los bosques (Paritsis et al., 2015; Pausas, 2015). Por lo tanto, tal como se ha sugerido para otros ecosistemas, es probable que estos tipos de vegetación representen estados alternativos mantenidos por distintos regímenes de fuego (Pausas, 2015).

Nuestros resultados indican que la composición de las comunidades de plantas es fuertemente afectada por el régimen de fuego, lo que se evidencia a partir de especies vegetales indicadoras exclusivas de sitios no quemados, es decir, que ocurren consistentemente en este tipo de escenarios. De acuerdo a estos resultados, las altas frecuencias de fuego generan un fuerte filtro ecológico en donde solo las especies tolerantes sobreviven y persisten (Pausas \& Verdú, 2008), y un subgrupo de éstas son las que ocurren en las comunidades sujetas a incendios, divergiendo florísticamente de las comunidades no quemadas. Es interesante destacar que la composición no fue diferente entre sitios con altas y bajas frecuencias de fuego, lo que indicaría que los intervalos de alrededor de 10 años entre eventos de fuego no son suficientes para recuperar especies indicadoras y así asemejar su composición a comunidades no quemadas. Asimismo, si bien la carga ganadera actual de los sitios no explicó las variaciones en la composición florística, el impacto relativo de la herbivoría puede ser diferente en sitios con diferente régimen de fuego. Posiblemente el efecto de una similar intensidad de ramoneo comprometa más seriamente la regeneración en sitios con alta frecuencia de fuego que en sitios con baja frecuencia o no quemados, impidiendo que se regeneren distintas especies y poniendo en riesgo la capacidad de resiliencia de estos ambientes.

Uno de los mecanismos por los que la frecuencia de fuego modifica la comunidad de plantas es a través del cambio estructural de la vegetación. Los cambios fisonómicos inducidos por la frecuencia de fuego sugieren que al no recuperarse la estructura arbórea se estaría afectando directamente la composición de especies. Además, estas comunidades estructuralmente diferentes podrían presentar divergencias funcionales en cuanto a rasgos de regeneración post-fuego (resistencia al fuego y reclutamiento de plántulas), de asimilación de recursos (Veldman et al., 2013; Carbone \& Aguilar, 2016), y posiblemente en las interacciones bióticas con animales y en su éxito reproductivo (Enright et al., 2015; Carbone, 2017). El escenario no quemado mostró especies indicadoras que típicamente se desarrollan en el sotobosque con una reducida intensidad lumínica (e.g., Lorentzianthus viscidus y Dicliptera squarrosa), o que necesitan en mayor medida de alguna especie leñosa como elemento de soporte para desarrollarse (e.g., Araujia odorata y Tillandsia capillaris). No obstante, la ocurrencia de especies indicadoras en escenarios sin fuego no necesariamente estaría relacionada con la falta de rasgos de tolerancia a disturbios (e.g., capacidad de rebrote). En este sentido, resulta contra intuitivo que el único árbol que resultó indicador de esta condición, Celtis ehrembergiana, presenta altos niveles de rebrote basal y supervivencia post-fuego (Herrero et al., 2016). Sin embargo, esta especie presenta una corteza delgada escasamente aislante del fuego (Kunst et al., 2014) y un extendido periodo juvenil hasta alcanzar edad reproductiva, los que podrían ser rasgos de sensibilidad a las elevadas frecuencias de fuego. Por otro lado, la única especie que resultó indicadora de ambientes quemados fue el arbusto perenne Abutilon pauciflorum, que presenta una elevada capacidad 
de rebrote y alta tolerancia a condiciones de estrés hídrico inducido por el fuego (Obs. pers). Estudios previos han observado la asociación de esta especie en comunidades herbáceas a la presencia de fuego (Sirolli \& Kalesnik, 2011; Carreras et al., 2012), sugiriendo que además de características morfológicas favorables para la regeneración post-incendio (presencia de xilopodio; Fuentes et al., 2011), su germinación también podría ser estimulada por el fuego (Galíndez et al., 2010). Resultaría interesante indagar sobre la ecología reproductiva y las interacciones bióticas de las especies indicadoras para tratar de explicar los mecanismos ecológicos de su ocurrencia diferencial en regímenes de fuego contrastantes.

La menor riqueza vegetal y el cambio en la composición de la vegetación en los ambientes con alta frecuencia de fuego, indican que el fuego es un fuerte proceso ecológico que estructura las comunidades, donde persisten aquellas especies con rasgos de historia de vida como el rebrote y el ciclo de vida que confieren a las plantas valor adaptativo ante un nuevo régimen de fuego (Pausas \& Verdú, 2008; Cianciaruso et al., 2012). Cuando las condiciones de un régimen de disturbio se tornan extremas, como es el caso de las elevadas frecuencias de fuego reportadas para las Sierras Chicas (Argañaraz, 2016), puede desencadenarse una homogeneización de la vegetación y pérdida de diversidad funcional, como ya se ha documentado para otras regiones del mundo con regímenes de fuego cambiantes (Li \& Waller, 2015). La homogeneización biótica se refiere a una reorganización no aleatoria del ensamblaje de especies, con incremento de la similitud genética, taxonómica o funcional como resultado de extinciones locales y de invasión de plantas exóticas (Olden et al., 2004; Rooney et al., 2007). Las especies que persisten bajo nuevos regímenes de fuego comparten atributos como el ciclo de vida, el tipo de polinización y el grado de tolerancia a las nuevas condiciones abióticas ( $\mathrm{Li}$ \& Waller, 2015). En los sitios con alta frecuencia de fuego, la persistencia de las especies se debe a la capacidad de rebrote de órganos vegetativos principalmente subterráneos (xilopodios, rizomas, raíces gemíferas, etc.) sumado a un corto periodo hasta alcanzar la edad reproductiva. Las plantas con sistemas radicales robustos y profundos pueden ser más tolerantes a las condiciones de sequía que generan los incendios (Bond \& van Wilgen, 1996). Sin embargo, otros procesos relacionados con la reproducción sexual y el reclutamiento pueden ser fuertemente afectados por la frecuencia de fuego (Enright et al., 2015). Al respecto se ha observado que las limitaciones edáficas impuestas por la frecuencia de fuego pueden limitar fuertemente la producción de frutos y semillas de hierbas perennes que rebrotan exitosamente en el Chaco Serrano (Carbone, 2017). Además, estas condiciones estresantes suelen ser un impedimento para el reclutamiento por semillas de muchas especies de plantas, en donde la mortalidad de plántulas suele ser elevada y restringida a períodos húmedos continuos sin fuegos. Visualizando el proceso de homogeneización biótica, los ambientes del Chaco Serrano con incendios periódicos podrían responder con un mayor predominio de especies rebrotadoras, con alta tolerancia a condiciones extremas de déficit hídrico e irradiación solar y con estrategias de aseguramiento reproductivo (i.e., independencia de polinizadores vía autogamia o cleistogamia; Carbone, 2017). Entre las consecuencias de una comunidad más homogénea se presentan una disminución general del funcionamiento ecosistémico, de los servicios ambientales que esta brinda y de su resiliencia debido al acotamiento del rango de respuestas de las especies que conforman la comunidad homogenizada (Olden et al., 2004). Sumado al fuego, otras presiones antrópicas como la pérdida y fragmentación del hábitat, la urbanización y el sobrepastoreo, las comunidades del Chaco Serrano se están convirtiendo en ambientes cada vez más hostiles que gradualmente conservarán menos especies, de grupos funcionales similares.

A la luz de establecer estrategias de conservación y manejo sustentable de los recursos naturales nativos, es necesario considerar la dinámica fuego-suelo-vegetación en el marco de las actividades socio-productivas de la región. Este trabajo sugiere que los esfuerzos deberían orientarse a conservar el acervo de especies de los sitios no quemados, monitorear la regeneración de los ambientes incendiados, manejar y regular la actividad ganadera (carga animal) de los sitios quemados en función del tiempo desde el último fuego y de su frecuencia, al mismo tiempo que restaurar los sitios degradados por fuegos recurrentes. 


\section{Agradecimientos}

Agradecemos a M. Giorgis y a J. P. Argañaraz por brindarnos la información geográfica de la historia de fuego de la región estudiada, a los propietarios de los campos por permitirnos realizar los muestreos, a M.C. Díaz Vélez por colaborar con los muestreos de campo, a M.P. Suárez Santillán por la ayuda en la identificación de las especies. Agradecemos a P. Jaureguiberry y a un revisor anónimo que con sus observaciones ayudaron a mejorar el manuscrito original. Este trabajo fue financiado por la Secretaría de Ciencia y TécnicaUNC (05/G457), CONICET (PIP 0019) y FONCyT (PICT 2011-1606). L.M.C. y N.A-A. son becarios posdoctorales, J.T. es becaria doctoral y R.A. es investigador adjunto del Consejo Nacional de Investigaciones Científicas y Técnicas (CONICET).

\section{Bibliografía}

ARGAÑARAZ, J. P. 2016. Dinámica espacial del fuego en las Sierras de Córdoba. Tesis Doctoral. FCEFyN, Universidad Nacional de Córdoba, Argentina. 217 p.

ARGAÑARAZ, J. P., G. G. PIZARRO, M. ZAK \& L. M. BELLIS. 2015 a. Fire regime, climate, and Vegetation in the Sierras de Córdoba, Argentina. Fire Ecology 11(1): 55-73.

ARGAÑARAZ, J. P., G. G. PIZARRO, M. ZAK, M. A. LANDI \& L. M. BELLIS. 2015 b. Human and biophysical drivers of fires in semiarid Chaco mountains of central Argentina. Sci. Total Environ. 520: 1-12.

BIRK, E. M. \& R. G. BRIDGES. 1989. Recurrent fires and fuel accumulation in even-aged Blackbutt (Eucalyptus pilularis) forests. For. Ecol. Manage. 29: 59-79.

BOND, W. J. \& J. J. MIDGLEY. 2001 Ecology of sprouting in woody plants: the persistence niche. Trends Ecol. Evol. 16:45-51.

BOND, W. J. \& B. W. VAN WILGEN. 1996. Fire and plants. Springer, Netherlands. $263 \mathrm{p}$.

BOND, W. J., F. I. WOODWARD \& G. F. MIDGLEY. 2005. The global distribution of ecosystems in a world without fire. New Phytol. 165(2): 525-538.

BRAUN-BLANQUET, J. 1950. Sociología vegetal: estudios de las comunidades vegetales. Buenos Aires: Acme. 444 p.

BRAVO, S., C. KUNST, A. GIMENEZ \& G. MOGLIA. 2001 Fire regime of a Elionorus muticus Spreng. savanna, western Chaco region, Argentina. Int. J. Wildland Fire 10: 65-72.
BRAVO, S., C. KUNST, R. GRAU \& E. ARÁOZ. 2010. Fire-rainfall relationships in Argentine Chaco savannas. J. Arid Environ. 74(10): 1319-1323.

BUCHER, E. 1982. Chaco and Caatinga. South American arid savannas, woodlands and thickets. In: Huntley, B. et al. (eds), Ecology of tropical savannas. SpringerVerlan Berlin.

BUCHER, E. H. \& P. C. HUSZAR. 1999. Sustainable management of the Gran Chaco of South America: ecological promise and economic constraints. $J$. Environ. Manag. 57(2): 99-108.

BUCHER, E. H. \& J. SCHOFIELD. 1981. Economic assault on Chagas disease. New Sci. 92: 321- 324.

CABIDO, M., M. L. CARRANZA, A. ACOSTA \& S. PÁEZ. 1991. Contribución al conocimiento fitosociológico del Bosque Chaqueño Serrano en la provincia de Córdoba, Argentina. Phytocoenologia 19: 547-566.

CABRERA, A. L. 1971 Fitogeografía de la República Argentina. Bol. Soc. Argen. Bot. 14 (1-2): 1-42.

CAPITANELLI, C. 1979. Clima. En: VÁZQUEZ, J. B., R. A. MIATELLO \& M. E. ROQUÉ (eds.), Geografia fisica de la provincia de Córdoba, pp. 45-138. Ed. Boldt. Argentina.

CARBONE. L. M. 2017. Ecología reproductiva de Fabaceae nativas forrajeras en diferentes escenarios post-fuego de las Sierras Chicas de Córdoba (Argentina). Tesis Doctoral. FCEFyN. Universidad Nacional de Córdoba, Argentina. $176 \mathrm{p}$.

CARBONE, L. M. \& R. AGUILAR. 2016. Contrasting effects of fire frequency on plant traits of three dominant perennial herbs from Chaco Serrano. Austral Ecol. 41(7): 778-790.

CARRERAS, M. E., E. FUENTES, J. E. MARTINAT \& L. M. CARBONE. 2012. Reconocimiento de diásporas de Malveae (Malvaceae) en muestras de suelos de zonas serranas (Sierras Chicas, Córdoba, Argentina) afectadas por incendios. Rodriguésia 63(3): 501-512.

CIANCIARUSO, M. V., I. A. SILVA, A M. A. BATALH, K. J. GASTON \& O. L. PETCHEY. 2012. The influence of fire on phylogenetic and functional structure of woody savannas: moving from species to individuals. Perspect. Plant. Ecol. 14: 205-216.

CLARKE, K. R. 1993. Non-parametric multivariate analysis of changes in community structure. Aust. $J$. Ecol. 18: 117-143.

COLWELL, R. K. 2013. EstimateS, Version 9.1: Statistical Estimation of Species Richness and Shared Species from Samples. User's Guide and application. Disponible en: http://purl.oclc.org/estimates. [Acceso: 20 octubre 2016].

COLLINS, S. L., S. M. GLENN \& D. J. GIBSON. 1995. Experimental analysis of intermediate disturbance and initial floristic composition: decoupling cause and effect. Ecology 76: 486-492. 
CONNELL, J. H. 1978. Diversity in tropical rain forests and coral reefs. Science 199: 1302-1310.

CHAO, A. \& L. JOST. 2012. Coverage $\square$ based rarefaction and extrapolation: standardizing samples by completeness rather than size. Ecology 93(12): 25332547.

CHAO, A., K. H. MA \& T. C. HSIEH. 2016. iNEXT (iNterpolation and EXTrapolation) Online. Program and User's Guide. Disponible en: http://chao.stat. nthu.edu.tw/wordpress/software_download/. [Acceso: 15 Mayo 2017].

DAVIES, G. M. 2013. Understanding fire regimes and the ecological effects of fire. En: Belcher, C. M. (Ed.). Fire phenomena and the earth system: an interdisciplinary guide to fire science, pp. 95-124. First Edition, John Wiley \& Sons Ltd. Published, UK.

DE CÁCERES, M. \& P. LEGENDRE. 2009. Associations between species and groups of sites: indices and statistical inference. Ecology 90(12): 3566-3574.

ENRIGHT, N. J., J. B. FONTAINE, D. M. BOWMAN, R. A. BRADSTOCK \& R. J. WILLIAMS. 2015. Interval squeeze: altered fire regimes and demographic responses interact to threaten woody species persistence as climate changes. Front. Ecol. Environ. 13(5): 265-272.

FUENTES E., M. E.CARRERAS, L. M. CARBONE, G. JEWSBURY, M. J. LOYOLA \& J. E. MARTINAT. 2011. Especies nativas de las Sierras Chicas (Córdoba, Argentina) con estrategias de regeneración post-fuego. Bol. Soc. Argen. Bot. 46 (supl): 192.

FUENTES E., M. E.CARRERAS, L. M. CARBONE, S.M. PONS, G. JEWSBURY, M. J. LOYOLA, J. E. MARTINAT \& M.A. CASTILLO MOINE. 2013. Escenarios florísticos post-fuego en las Sierras Chicas de Córdoba. Bol. Soc. Argen. Bot. 48 (supl): 63-64.

GALÍNDEZ, G., P. ORTEGA-BAES, C. E. SEAL, M. I. DAWS, A. L. SCOPEL \& H. W. PRITCHARD. 2010. Physical seed dormancy in Collaea argentina (Fabaceae) and Abutilon pauciflorum (Malvaceae) after 4 years storage. Seed Science and Technology 38(3): 777-782.

GARREAUD, R. D., M. G. NICORA, R. E. BÜRGESSER \& E. E. ÁVILA. 2014. Lightning in western Patagonia. J. Geophys Res-Atmos. 119(8): 44714485.

GAVIER, G. I. \& E. H. BUCHER. 2004. Deforestación de las Sierras Chicas de Córdoba (Argentina) en el período 1970-1997. Academia Nacional de Ciencias. Miscelánea 101:1-27.

GIORGIS, M. A. 2011. Caracterización florística $y$ estructural del Bosque Chaqueño Serrano (Córdoba) en relación a gradientes ambientales y de uso. Tesis Doctoral. FCEFyN, Universidad Nacional de Córdoba, Argentina. 174 p.
GIORGIS, M. A. \& P. A. TECCO. 2014. Árboles y arbustos invasores de la Provincia de Córdoba (Argentina): una contribución a la sistematización de bases de datos globales. Bol. Soc. Argen. Bot. 49(4): 581-603.

GIORGIS, M. A., A. M. CINGOLANI \& M. CABIDO. 2013. El efecto del fuego y las características topográficas sobre la vegetación y las propiedades del suelo en la zona de transición entre bosques y pastizales de las sierras de Córdoba, Argentina. Bol. Soc. Argen. Bot. 48(3-4): 493-513.

GOVENDER, N., W. S. W. TROLLOPE \& B. W. VAN WILGEN. 2006. The effect of ire season, fire frequency, rainfall and management on fire intensity in savanna vegetation in South Africa. J. Appl. Ecol. 43: 748-758.

HERRERO, M. L., R. C. TORRES \& D. RENISON. 2016. Do wildfires promote woody species invasion in a fireadapted ecosystem? Post-fire resprouting of native and non-native woody plants in central Argentina. Environ. Manage. 57(2): 308-317.

HOBBS, R.J., A. U. MALLIK \& C. H. GIMINGHAM. 1984. Studies on fire in Scottish heathland communities: III. Vital attributes of the species. J. Ecol. 72: 963-976.

HUSTON, M. A. 1979. A general hypothesis of species diversity. Am. Nat. 113: 81-101.

JAUREGUIBERRY, P. 2012. Caracteres funcionales, flamabilidad y respuesta al fuego de especies vegetales dominantes en distintas situaciones de uso de la tierra en el centro-oeste de Argentina. Tesis Doctoral. FCEFyN, Universidad Nacional de Córdoba, Argentina. 203 p.

KEELEY, J. E., J. G. PAUSAS, P. W. RUNDEL, W. J. BOND \& R. A. BRADSTOCK. 2011. Fire as an evolutionary pressure shaping plant traits. Trends Plant Sci. 16(8): 406-411.

KUNST, C. 2011. Ecología y uso del fuego en la región chaqueña Argentina. Boletín Informativo CIDEU (10): 81-105.

KUNST, C. \& S. BRAVO. 2003. Ecología y régimen de fuego en la región chaqueña argentina. En: Kunst, C. R. et al. (eds.), Fuego en los ecosistemas Argentinos, pp. 47-59. INTA, Santiago del Estero, Arg.

KUNST, C., S. BRAVO, F. MOSCOVICH, J. HERRERA, J.GODOY \& S. VÉLEZ. 2003. Fecha de aplicación de fuego y diversidad de herbáceas en una sabana de Elionorus muticus (Spreng) O. Kuntze. Rev. Chil. Hist. Nat. 76(1): 105-115.

KUNST, C., S. BRAVO, R. LEDESMA, M. NAVALL, A. ANRÍQUEZ, D. CORIA, J. SILBERMAN, A. GÓMEZ \& A. ALBANESI. 2014. Ecology and management of the dry forests and savannas of the western Chaco region, Argentina. En: Greer, F. E. (Ed), Dry forest, pp: 132-163. Nova Science Publishers.

LI, D. \& D. WALLER. 2015. Drivers of observed biotic homogenization in pine barrens of central Wisconsin. Ecology 96(4): 1030-1041. 
LI, W., X. ZUO \& J. M. KNOPS. 2013. Different fire frequency impacts over 27 years on vegetation succession in an infertile old-field grassland. Rangeland Ecol. Manage. 66(3): 267-273.

LUNT, I. D. \& J. W. MORGAN. 2002. The role of fire regimes in temperate lowland Grasslands of southeastern Australia. In: Bradstock, R. A., J. E. Williams, A. M. Gill, (eds.), Flammable Australia: The Fire Regimes and Biodiversity of a Continent, pp. 177-196. Cambridge University Press, Cambridge.

LUTI, R., M. A. BERTRAN DE SOLIS, M. F. GALERA, N. MÜLLER DE FERREIRA, M. BERZAL, M. NORES, M. A. HERRERA \& J. C. BARRERA. 1979. Vegetación. En: VÁZQUEZ , J. B., R. A. MIATELLO \& M. E. ROQUÉ (eds.), Geografía física de la provincia de Córdoba, pp. 297-368. Editorial Boldt, Buenos Aires, Arg.

MANDAI, C. Y. 2015. Computer simulation in ecology: an intuitive interface between verbal and mathematical models. PhD Thesis. $97 \mathrm{p}$.

MIGLIETTA, S. 1994. Patrón de ocurrencia de incendios y su efecto sobre la vegetación en el Bosque Serrano de Córdoba. Tesis de Maestría. FCEFyN, Universidad Nacional de Córdoba, Argentina. 60 p.

MORELLO, J. \& C. SARAVIA TOLEDO. 1959. El bosque chaqueño I y II. Revista Agronómica del Noroeste Argentino 3: 5-81/209-258.

NAYAK, R. R., S. VAIDYANATHAN \& J. KRISHNASWAMY. 2014. Fire and grazing modify grass community response to environmental determinants in savannas: Implications for sustainable use. Agric. Ecosyst. Environ. 185: 197-207.

NICORA, M. G., E. J. QUEL, R. E. BURGESSER \& E. E. AVILA. 2013. Electric atmospheric activity in Argentina, a study for estimating the annual death rate by lightning. International Symposium on Lightning Protection (XII SIPDA), Belo Horizonte, Brazil. pp. 340-344.

OKSANEN, J., F. G. BLANCHET. M. FRIENDLY, et al. 2016. vegan: Community Ecology Package. R package version 2.4-0. Disponible en: https://cran.rproject.org/web/packages/vegan/index.html

OLDEN, J. D., N. L. POFF, M. R. DOUGLAS, M. E. DOUGLAS \& K. D. FAUSCH. 2004. Ecological and evolutionary consequences of biotic homogenization. Trends Ecol. Evol. 19(1): 18-24.

PARITSIS, J., T. T. VEBLEN \& A. HOLZ. 2015. Positive fire feedbacks contribute to shifts from Nothofagus pumilio forests to fire $\square$ prone shrublands in Patagonia. J. Veg. Sci. 26: 89-101.

PAUSAS, J. G. 2012. Incendios Forestales. Ed. Catarata. CSIC. Madrid, España. 119 p.

PAUSAS, J. G. 2015. Alternative fire-driven vegetation states. J. Veg. Sci. 26: 4-6.
PAUSAS, J. G. \& M. VERDÚ. 2008. Fire reduces morphospace occupation in plant communities. Ecology 89(8): 2181-2186.

PAUSAS, J. G. \& J. E. KEELEY. 2009. A burning story: the role of fire in the history of life. BioScience 59(7): 598-601.

PAUSAS, J. G. \& E. RIBEIRO. 2017. Fire and plant diversity at the global scale. Global Ecol. Biogeogr.: 26: 889-897.

PNEF (Programa Nacional de Estadística Forestal). 2011. Estadística de Incendios Forestales 2010. Secretaría de Ambiente y Desarrollo Sustentable de la Nación Argentina. Buenos Aires, Arg. 102p. ISSN 1850-7239.

R CORE TEAM. 2016. R: A language and environment for statistical computing. R Foundation for Statistical Computing, Vienna, Austria. ISBN 3-900051-07-0, Disponible en: http://www.R-project.org/.

RENISON, D., A. M. CINGOLANI \& R. SUAREZ. 2002. Efectos del fuego sobre un bosquecillo de Polylepis australis (Rosaceae) en las montañas de Córdoba, Argentina. Rev. Chil. Hist. Nat. 75(4): 719-727.

ROONEY, T. P., J. D. OLDEN, M. K.LEACH \& D. A. ROGERS. 2007. Biotic homogenization and conservation prioritization. Biol. Conserv. 134(3): 447-450.

SIROLLI, H. \& F. A. KALESNIK. 2011. Effects of fire on a forest-grassland ecotone in De La Plata River, Argentina. Plant Ecol. 212(4): 689-700.

TORRES, R. C., M. A. GIORGIS, C. TRILlO, L. VOLKMANN, P. DEMAIO, J. HEREDIA \& D. RENISON. 2014. Post-fire recovery occurs overwhelmingly by resprouting in the Chaco Serrano forest of Central Argentina. Austral Ecol. 39(3): 346-354.

UYS, R. G., W. J. BOND \& T. M. EVERSON. 2004. The effect of different fire regimes on plant diversity in southern African grasslands. Biol. Conserv. 118(4): 489-499.

VELDMAN, J. W., W. B. MATTINGLY \& L. A. BRUDVIG. 2013. Understory plant communities and the functional distinction between savanna trees, forest trees, and pines. Ecology 94(2): 424434.

VERZINO, G., J. JOSEAU, M.DORADO, E.GELLERT, S.RODRÍGUEZ REARTES \& R. NÓBILE. 2005. Impacto de los incendios sobre la diversidad vegetal, Sierras de Córdoba, Argentina. Ecología Aplicada 4(1-2): 25-34.

WATSON, P. J., R. A. BRADSTOCK \& E. C. MORRIS. 2009. Fire frequency influences composition and structure of the shrub layer in an Australian subcoastal temperate grassy woodland. Austral Ecol. 34(2): 218-232. 


\section{M. Carbone et al. - Cambios florísticos inducidos por el fuego}

WILLIAMS, R. J., G. D. COOK, A. M. GILL \& P. H. R. MOORE. 1999). Fire regime, fire intensity and tree survival in a tropical savanna in northern Australia. Aust. J. Ecol. 24: 50-59.

ZULOAGA, F.O., O. MORRONE \& M. J. BELGRANO. 2008. Catálogo de Plantas Vasculares del Cono Sur (Argentina, Sur de Brasil, Chile, Paraguay y Uruguay). Monograph of Systematic Botany Missouri Botanical Garden. Missouri Botanical Garden Press, Saint Louis.

Recibido el 25 de marzo de 2017, aceptado el 4 de agosto de 2017. 
Apéndice 1. Composición florística de los escenarios con diferente frecuencia de fuego del Chaco Serrano. Se detalla para cada taxón el origen: nativo (N), endémico (End), exótico (E) y cosmopolita $(C)$; la forma de crecimiento: árbol $(A)$, arbusto (a), enredadera $(E)$, hierba $(H)$ y graminoide $(G)$; y el porcentaje de frecuencia relativa del total de censos en el que fue registrado en cada escenario de frecuencia de fuego: no quemado, baja y alta frecuencia de fuego. El símbolo + en frecuencia relativa indica los casos particulares en los que un individuo aislado de un determinado taxón fue registrado en un solo sitio, con una cobertura despreciable. El asterisco $\left(^{*}\right)$ indica un nuevo registro del taxón para la flora del centro del país (Córdoba).

Base de datos para la actualización de los nombres científicos:

- Catálogo de las Plantas Vasculares del Cono Sur (Instituto de Botánica Darwinion, Argentina). Acceso el 30 de abril de 2017 . http://www.darwin.edu.ar/Proyectos/FloraArgentina/fa.htm

-Tropicos.org. Missouri Botanical Garden. Acceso: 30 de abril de 2017. http://www.tropicos.org

\begin{tabular}{|c|c|c|c|c|c|c|}
\hline Taxón & Familia & Origen & $\begin{array}{l}\text { Forma de } \\
\text { crecimiento }\end{array}$ & $\begin{array}{c}\text { No } \\
\text { Quemado }\end{array}$ & $\begin{array}{c}\text { Baja } \\
\text { frecuencia } \\
\text { de fuego }\end{array}$ & $\begin{array}{c}\text { Alta } \\
\text { frecuencia } \\
\text { de fuego }\end{array}$ \\
\hline Abutilon grandifolium & Malvaceae & $\mathrm{N}$ & a & 33 & 0 & 0 \\
\hline Abutilon pauciflorum & Malvaceae & $\mathrm{N}$ & a & 0 & 67 & 100 \\
\hline Abutilon terminale & Malvaceae & End & a & 0 & 0 & 33 \\
\hline Acacia caven & Fabaceae & N & A & 100 & 100 & 67 \\
\hline Acacia praecox & Fabaceae & $\mathrm{N}$ & A & 33 & 0 & 0 \\
\hline Acalypha communis & Euphorbiaceae & $\mathrm{N}$ & $\mathrm{H}$ & 100 & 100 & 100 \\
\hline Acalypha poiretii & Euphorbiaceae & N & $\mathrm{H}$ & + & 0 & 0 \\
\hline Acanthostyles buniifolius & Asteraceae & N & a & 33 & 67 & 33 \\
\hline Acer negundo & Aceraceae & $\mathrm{E}$ & A & + & 0 & 0 \\
\hline Achyrocline flaccida & Asteraceae & N & $\mathrm{H}$ & 67 & + & + \\
\hline Achyrocline venosa & Asteraceae & $\mathrm{N}$ & $\mathrm{H}$ & 67 & + & 67 \\
\hline Adesmia inacana var. incana & Fabaceae & End & $\mathrm{H}$ & + & 33 & 0 \\
\hline $\begin{array}{l}\text { Aeschynomene histrix } \\
\text { var. inacana }\end{array}$ & Fabaceae & $\mathrm{N}$ & $\mathrm{H}$ & 0 & 33 & 0 \\
\hline $\begin{array}{l}\text { Agrostis montevidensis } \\
\text { f. montevidensis }\end{array}$ & Poaceae & G & G & 67 & 67 & 100 \\
\hline Aloysia gratissima & Verbenaceae & $\mathrm{N}$ & a & 100 & 33 & 67 \\
\hline $\begin{array}{l}\text { Alternanthera paronychioides } \\
\text { ssp. chacoensis }\end{array}$ & Amaranthaceae & $\mathrm{N}$ & $\mathrm{H}$ & 33 & + & 0 \\
\hline $\begin{array}{l}\text { Amaranthus hybridus } \\
\text { ssp. hybridus }\end{array}$ & Amaranthaceae & $\mathrm{E}$ & $\mathrm{H}$ & + & 0 & 33 \\
\hline Amphilophium carolinae & Bignoniaceae & $\mathrm{N}$ & E & 67 & 0 & 0 \\
\hline Anemia tormentosa & Anemiaceae & $\mathrm{N}$ & $\mathrm{H}$ & 67 & 67 & 33 \\
\hline Anoda cristata & Malvaceae & $\mathrm{N}$ & H & + & 33 & 0 \\
\hline Anredera cordifolia & Basellaceae & $\mathrm{N}$ & $\mathrm{E}$ & 100 & 67 & 67 \\
\hline Araujia odorata & Apocynaceae & $\mathrm{N}$ & E & 100 & 0 & 0 \\
\hline Aristida adscensionis & Poaceae & G & G & 0 & 33 & 0 \\
\hline Aristida spegazzinii & Poaceae & G & G & 0 & 33 & 0 \\
\hline Artemisia annua & Asteraceae & E & $\mathrm{H}$ & 0 & 0 & 67 \\
\hline Asclepias mellodora & Apocynaceae & N & $\mathrm{H}$ & + & 0 & + \\
\hline Ayenia cordobensis & Malvaceae & End & $\mathrm{H}$ & + & 67 & 0 \\
\hline
\end{tabular}


L. M. Carbone et al. - Cambios florísticos inducidos por el fuego

\begin{tabular}{|c|c|c|c|c|c|c|}
\hline Taxón & Familia & Origen & $\begin{array}{l}\text { Forma de } \\
\text { crecimiento }\end{array}$ & $\begin{array}{c}\text { No } \\
\text { Quemado }\end{array}$ & $\begin{array}{c}\text { Baja } \\
\text { frecuencia } \\
\text { de fuego }\end{array}$ & $\begin{array}{c}\text { Alta } \\
\text { frecuencia } \\
\text { de fuego }\end{array}$ \\
\hline Baccharis aliena & Asteraceae & End & a & 33 & 33 & 33 \\
\hline Baccharis coridifolia & Asteraceae & $\mathrm{N}$ & a & 100 & 100 & 33 \\
\hline Baccharis crispa & Asteraceae & $\mathrm{N}$ & a & 33 & 33 & 0 \\
\hline Baccharis glutinosa & Asteraceae & $\mathrm{N}$ & $\mathrm{H}$ & + & 0 & 0 \\
\hline Baccharis linearifolia & Asteraceae & $\mathrm{N}$ & a & 33 & 100 & 67 \\
\hline Bernardia multicaulis & Euphorbiaceae & $\mathrm{N}$ & $\mathrm{H}$ & 33 & 67 & 67 \\
\hline $\begin{array}{l}\text { Bidens subalternans } \\
\text { var. subalternans }\end{array}$ & Asteraceae & $\mathrm{N}$ & $\mathrm{H}$ & 67 & 100 & 100 \\
\hline Blechnum laevigatum & Blechnaceae & $\mathrm{N}$ & $\mathrm{H}$ & + & 0 & 33 \\
\hline Borreria eryngioides var. ostenii & Rubiaceae & End & $\mathrm{H}$ & 0 & 67 & + \\
\hline Borreria spinosa & Rubiaceae & N & $\mathrm{H}$ & 67 & 100 & 33 \\
\hline Bothriochloa alta & Poaceae & N & G & 33 & 100 & 67 \\
\hline Bothriochloa barbinodis & Poaceae & N & G & 33 & 33 & 33 \\
\hline Bothriochloa laguroides & Poaceae & $\mathrm{N}$ & G & + & 0 & 0 \\
\hline Bougainvillea stipitata & Nyctaginaceae & $\mathrm{N}$ & A & 33 & 0 & 0 \\
\hline $\begin{array}{l}\text { Bouteloua curtipendula } \\
\text { var caespitosa }\end{array}$ & Poaceae & N & G & 100 & 100 & 100 \\
\hline Bouteloua megapotamica & Poaceae & N & G & + & 0 & 0 \\
\hline $\begin{array}{l}\text { Bromus catharticus } \\
\text { var. catharticus }\end{array}$ & Poaceae & N & G & 0 & 0 & + \\
\hline $\begin{array}{l}\text { Bulbostylis juncoides } \\
\text { var. juncoides }\end{array}$ & Cyperaceae & $\mathrm{N}$ & $\mathrm{H}$ & + & 33 & 0 \\
\hline Caesalpinia gilliesii & Fabaceae & $\mathrm{N}$ & a & + & 0 & 0 \\
\hline Cantinoa mutabilis & Lamiaceae & $\mathrm{N}$ & $\mathrm{H}$ & 100 & 100 & 100 \\
\hline Cardiospermum halicacabum & Sapindaceae & C & E & 33 & 0 & 0 \\
\hline Carduus acanthoides & Asteraceae & $E$ & $\mathrm{H}$ & + & 0 & 0 \\
\hline Celtis australis & Celtidaceae & E & A & 33 & 33 & 0 \\
\hline Celtis ehrenbergiana & Celtidaceae & N & A & 100 & + & + \\
\hline Cenchrus myosuroides & Poaceae & $\mathrm{N}$ & G & 0 & + & 0 \\
\hline Cenchrus spinifex & Poaceae & $\mathrm{N}$ & G & + & 0 & 0 \\
\hline Centrosema virginianum & Fabaceae & N & E & 67 & 33 & 33 \\
\hline Cestrum parqui & Solanaceae & N & a & 67 & 67 & 67 \\
\hline Chaptalia integerrima & Asteraceae & N & $\mathrm{H}$ & 100 & 100 & 67 \\
\hline Chascolytrum subaristatum & Poaceae & N & G & + & 0 & 0 \\
\hline Cheilanthes buchtienii & Pteridaceae & N & $\mathrm{H}$ & 0 & 100 & 33 \\
\hline Chenopodium album & Chenopodiaceae & E & $\mathrm{H}$ & + & 0 & 0 \\
\hline Chiropetalum griseum & Euphorbiaceae & N & a & 33 & + & 0 \\
\hline Chromolaena arnottiana & Asteraceae & $\mathrm{N}$ & a & 100 & + & 0 \\
\hline Chromolaena hirsuta & Asteraceae & End & $\mathrm{H}$ & 0 & 33 & 0 \\
\hline $\begin{array}{l}\text { Chrysanthellum indicum } \\
\text { ssp. afroamericanum }\end{array}$ & Asteraceae & $\mathrm{N}$ & $\mathrm{H}$ & 0 & 67 & 33 \\
\hline
\end{tabular}


Bol. Soc. Argent. Bot. 52 (4) 2017

\begin{tabular}{|c|c|c|c|c|c|c|}
\hline Taxón & Familia & Origen & $\begin{array}{l}\text { Forma de } \\
\text { crecimiento }\end{array}$ & $\begin{array}{c}\text { No } \\
\text { Quemado }\end{array}$ & $\begin{array}{c}\text { Baja } \\
\text { frecuencia } \\
\text { de fuego }\end{array}$ & $\begin{array}{c}\text { Alta } \\
\text { frecuencia } \\
\text { de fuego }\end{array}$ \\
\hline $\begin{array}{l}\text { Clematis montevidiensis } \\
\text { var. montevidensis }\end{array}$ & Ranunculaceae & $N$ & E & + & 0 & 0 \\
\hline Clitoria cordobensis & Fabaceae & End & $\mathrm{H}$ & + & 33 & + \\
\hline Colletia spinosissima & Rhamnaceae & $\mathrm{N}$ & a & 100 & 100 & 100 \\
\hline Cologania broussonetii & Fabaceae & $\mathrm{N}$ & E & 100 & 100 & 100 \\
\hline Commelina erecta & Commelinaceae & $N$ & $\mathrm{H}$ & 100 & 67 & 100 \\
\hline Commelina tuberosa & Commelinaceae & $\mathrm{N}$ & $\mathrm{H}$ & 33 & 0 & 0 \\
\hline Condalia buxifolia & Rhamnaceae & $\mathrm{N}$ & A & 100 & 67 & 67 \\
\hline Condalia microphylla & Rhamnaceae & End & a & 33 & 0 & 0 \\
\hline Condalia montana & Rhamnaceae & End & a & 33 & 0 & 0 \\
\hline Cortaderia selloana & Poaceae & $\mathrm{N}$ & G & + & 0 & + \\
\hline Cotoneaster pannosus & Rosaceae & $E$ & a & 33 & + & 0 \\
\hline Croton argentinus & Euphorbiaceae & End & a & 0 & 67 & 0 \\
\hline Croton hirtus & Euphorbiaceae & $N$ & $\mathrm{H}$ & 100 & + & 0 \\
\hline Croton lachnostachyus & Euphorbiaceae & $N$ & a & 67 & 0 & + \\
\hline Croton lanatus var. Iorentzii & Euphorbiaceae & End & a & 0 & 33 & 0 \\
\hline Croton serratifolius & Euphorbiaceae & N & a & 0 & 67 & 67 \\
\hline Croton subpannosus & Euphorbiaceae & End & a & 0 & 33 & 0 \\
\hline Cuphea glutinosa & Lythraceae & N & $\mathrm{H}$ & 67 & 67 & + \\
\hline Cynoglossum amabile & Boraginaceae & E & $\mathrm{H}$ & + & + & 0 \\
\hline Cyperus aggregatus & Cyperaceae & N & G & 100 & 67 & 100 \\
\hline Cyperus hermaphroditus & Cyperaceae & N & G & 67 & 33 & 33 \\
\hline Cyperus incomtus var. incomtus & Cyperaceae & $\mathrm{N}$ & G & 33 & 67 & 0 \\
\hline Dalea elegans var. elegans & Fabaceae & End & $\mathrm{H}$ & 0 & 33 & 0 \\
\hline $\begin{array}{l}\text { Desmanthus tatuhyensis } \\
\text { var. brevipes }\end{array}$ & Fabaceae & $\mathrm{N}$ & $\mathrm{H}$ & 0 & 100 & + \\
\hline Desmodium uncinatum & Fabaceae & N & $\mathrm{H}$ & 100 & 100 & 100 \\
\hline $\begin{array}{l}\text { Deyeuxia viridiflavescens } \\
\text { var. viridiflavescens }\end{array}$ & Poaceae & $\mathrm{N}$ & G & 0 & 67 & 33 \\
\hline Dichondra microcalyx & Convolvulaceae & N & $\mathrm{H}$ & 67 & 0 & 33 \\
\hline Dichondra sericea var. sericea & Convolvulaceae & $N$ & $\mathrm{H}$ & 67 & 67 & 67 \\
\hline Dicliptera squarrosa & Acanthaceae & N & $\mathrm{H}$ & 100 & + & 0 \\
\hline Digitaria eriantha & Poaceae & $E$ & G & 33 & 0 & 0 \\
\hline Digitaria sanguinalis & Poaceae & E & G & 33 & 0 & 0 \\
\hline Ditassa burchellii & Apocynaceae & N & E & + & 0 & 0 \\
\hline Dolichandra cynanchoides & Bignoniaceae & $\mathrm{N}$ & E & 67 & 0 & 0 \\
\hline Dolichandra unguis-cati & Bignoniaceae & N & E & + & 0 & 0 \\
\hline Dyschoriste humilis & Acanthaceae & $\mathrm{N}$ & $\mathrm{H}$ & 33 & 0 & 0 \\
\hline Echinopsis aurea & Cactaceae & End & $\mathrm{H}$ & + & 67 & 0 \\
\hline
\end{tabular}


L. M. Carbone et al. - Cambios florísticos inducidos por el fuego

\begin{tabular}{|c|c|c|c|c|c|c|}
\hline Taxón & Familia & Origen & $\begin{array}{l}\text { Forma de } \\
\text { crecimiento }\end{array}$ & $\begin{array}{c}\text { No } \\
\text { Quemado }\end{array}$ & $\begin{array}{c}\text { Baja } \\
\text { frecuencia } \\
\text { de fuego }\end{array}$ & $\begin{array}{c}\text { Alta } \\
\text { frecuencia } \\
\text { de fuego }\end{array}$ \\
\hline Eragrostis curvula & Poaceae & E & G & 0 & 0 & 67 \\
\hline Eragrostis polytricha & Poaceae & $\mathrm{N}$ & G & + & 67 & 33 \\
\hline Eryngium elegans & Apiaceae & $\mathrm{N}$ & $\mathrm{H}$ & 33 & + & 0 \\
\hline Eryngium horridum & Apiaceae & $\mathrm{N}$ & $\mathrm{H}$ & 67 & 100 & 100 \\
\hline Euphorbia acerensis & Euphorbiaceae & $\mathrm{N}$ & $\mathrm{H}$ & 100 & 0 & 0 \\
\hline Euphorbia hirta var. ophthalmica & Euphorbiaceae & E & $\mathrm{H}$ & 67 & 0 & 0 \\
\hline Euphorbia lorentzii & Euphorbiaceae & $\mathrm{N}$ & $\mathrm{H}$ & 67 & 100 & 33 \\
\hline Euphorbia pentadactyla & Euphorbiaceae & $\mathrm{N}$ & $\mathrm{H}$ & 67 & 100 & 33 \\
\hline Eustachys distichophylla & Poaceae & $\mathrm{N}$ & G & 0 & 0 & 67 \\
\hline Eustachys retusa & Poaceae & $\mathrm{N}$ & G & 67 & 100 & + \\
\hline Evolvulus sericeus var elongatus & Convolvulaceae & End & $\mathrm{H}$ & 0 & 33 & 0 \\
\hline Evolvulus sericeus var sericeus & Convolvulaceae & N & $\mathrm{H}$ & 33 & 100 & 0 \\
\hline Exhalimolobos weddellii & Brassicaceae & $\mathrm{N}$ & $\mathrm{H}$ & + & 0 & 33 \\
\hline Fleischmannia prasiifolia & Asteraceae & N & a & 33 & 0 & 0 \\
\hline $\begin{array}{l}\text { Gaillardia megapotamica } \\
\text { var. radiata }\end{array}$ & Asteraceae & End & $\mathrm{H}$ & + & 0 & 0 \\
\hline Galactia glaucophylla & Fabaceae & End & $\mathrm{H}$ & 0 & 33 & 0 \\
\hline Galactia marginalis & Fabaceae & N & $\mathrm{H}$ & 0 & 100 & 0 \\
\hline Galium latoramosum & Rubiaceae & N & $\mathrm{H}$ & 67 & 0 & + \\
\hline $\begin{array}{l}\text { Galium richardianum } \\
\text { ssp. richardianum }\end{array}$ & Rubiaceae & End & $\mathrm{H}$ & 0 & 67 & 0 \\
\hline Gaya parviflora & Malvaceae & N & $\mathrm{H}$ & 67 & 33 & 100 \\
\hline Glandularia peruviana & Verbenaceae & N & $\mathrm{H}$ & 33 & 67 & 67 \\
\hline Glandularia tenera & Verbenaceae & N & $\mathrm{H}$ & 0 & + & 0 \\
\hline Gleditsia triacanthos & Fabaceae & $E$ & A & + & 0 & 0 \\
\hline Gomphrena perennis & Amaranthaceae & $\mathrm{N}$ & $\mathrm{H}$ & 100 & 67 & 33 \\
\hline Gouinia latifolia & Poaceae & $\mathrm{N}$ & G & 33 & 0 & 0 \\
\hline Grindelia pulchella var. discoidea & Asteraceae & End & a & + & 0 & 0 \\
\hline Gymnocalycium erinaseum & Cactaceae & End & $\mathrm{H}$ & + & 0 & 0 \\
\hline Gymnocalycium monvillei & Cactaceae & End & $\mathrm{H}$ & + & 0 & 0 \\
\hline Gymnocalycium mostii & Cactaceae & End & $\mathrm{H}$ & 33 & 100 & 33 \\
\hline Gymnocalycium sp. & Cactaceae & End & $\mathrm{H}$ & 0 & 33 & 0 \\
\hline Gyptis artemisifolia & Asteraceae & End & $\mathrm{H}$ & 0 & 67 & 0 \\
\hline Heimia salicifolia & Lythraceae & N & a & 0 & + & + \\
\hline Heliotropium nicotianaefolium & Boraginaceae & N & $\mathrm{H}$ & + & + & 0 \\
\hline Heterosperma ovatifolium & Asteraceae & $\mathrm{N}$ & $\mathrm{H}$ & 100 & + & 33 \\
\hline Ipomoea hieronymi & Convolvulaceae & End & E & 0 & 67 & 33 \\
\hline Ipomoea purpurea & Convolvulaceae & $\mathrm{N}$ & $E$ & 67 & 0 & 0 \\
\hline Ipomoea rubriflora & Convolvulaceae & $\mathrm{N}$ & E & + & + & 0 \\
\hline
\end{tabular}


Bol. Soc. Argent. Bot. 52 (4) 2017

\begin{tabular}{|c|c|c|c|c|c|c|}
\hline Taxón & Familia & Origen & $\begin{array}{l}\text { Forma de } \\
\text { crecimiento }\end{array}$ & $\begin{array}{c}\text { No } \\
\text { Quemado }\end{array}$ & $\begin{array}{c}\text { Baja } \\
\text { frecuencia } \\
\text { de fuego }\end{array}$ & $\begin{array}{c}\text { Alta } \\
\text { frecuencia } \\
\text { de fuego }\end{array}$ \\
\hline Iresine diffusa & Amaranthaceae & $\mathrm{N}$ & $\mathrm{H}$ & 100 & 67 & 100 \\
\hline Janusia guaranitica & Malpighiaceae & $\mathrm{N}$ & E & 100 & + & 0 \\
\hline Jarava ichu & Poaceae & $\mathrm{N}$ & G & 33 & 0 & 0 \\
\hline Jarava pseudoichu & Poaceae & $\mathrm{N}$ & G & 100 & 33 & 33 \\
\hline Jodina rhombifolia & Cervantesiaceae & $\mathrm{N}$ & A & 0 & 0 & 33 \\
\hline Kageneckia lanceolata & Rosaceae & $\mathrm{N}$ & A & + & 0 & 0 \\
\hline Krapovickasia flavescens & Malvaceae & $\mathrm{N}$ & $\mathrm{H}$ & 67 & 100 & 100 \\
\hline Lantana camara & Verbenaceae & $\mathrm{N}$ & a & 67 & 0 & 67 \\
\hline Lantana grisebachii & Verbenaceae & $\mathrm{N}$ & a & 67 & + & 67 \\
\hline Lepechinia floribunda & Lamiaceae & $\mathrm{N}$ & a & 100 & 67 & 67 \\
\hline $\begin{array}{l}\text { Lessingianthus mollissimus } \\
\text { var. mollisimus }\end{array}$ & Asteraceae & End & $\mathrm{H}$ & 67 & 100 & 67 \\
\hline Ligaria cuneifolia & Loranthaceae & $\mathrm{N}$ & $\mathrm{H}$ & 33 & 0 & 0 \\
\hline Ligustrum lucidum & Oleaceae & $\mathrm{E}$ & A & + & 0 & 0 \\
\hline Lippia junelliana & Verbenaceae & End & a & 33 & 0 & 0 \\
\hline Lippia turbinata f. turbinata & Verbenaceae & $\mathrm{N}$ & a & 33 & 0 & 33 \\
\hline Lithraea molleoides & Anacardiaceae & $\mathrm{N}$ & A & 100 & 67 & 33 \\
\hline Lonicera japonica & Caprifoliaceae & $\mathrm{E}$ & E & + & 0 & 0 \\
\hline Lorentzianthus viscidus & Asteraceae & N & a & 100 & 0 & 0 \\
\hline Lucilia acutifolia & Asteraceae & $\mathrm{N}$ & $\mathrm{H}$ & 0 & 67 & + \\
\hline Malvastrum coromandelianum & Malvaceae & $\mathrm{N}$ & $\mathrm{H}$ & + & 0 & 0 \\
\hline Malvastrum interruptum & Malvaceae & $\mathrm{N}$ & $\mathrm{H}$ & 33 & 33 & 33 \\
\hline Mandevilla pentlandiana & Apocynaceae & N & E & 67 & + & 0 \\
\hline Mandevilla petraea & Apocynaceae & $\mathrm{N}$ & $\mathrm{H}$ & 33 & 33 & 0 \\
\hline Margyricarpus pinnatus & Rosaceae & N & $\mathrm{H}$ & + & 67 & 0 \\
\hline Matelea australis & Apocynaceae & End & $\mathrm{H}$ & 0 & 67 & + \\
\hline Melica macra & Poaceae & End & G & + & + & 0 \\
\hline Melinis repens & Poaceae & $\mathrm{E}$ & G & + & 0 & 0 \\
\hline Melochia argentina & Malvaceae & $\mathrm{N}$ & H & + & 0 & 0 \\
\hline Mentzelia scabra ssp. cordobensis & Loasaceae & End & a & 33 & 33 & 0 \\
\hline Minthostachys verticillata & Lamiaceae & End & $\mathrm{H}$ & 33 & 0 & 33 \\
\hline Mitracarpus megapotamicus & Rubiaceae & $\mathrm{N}$ & $\mathrm{H}$ & 0 & 67 & + \\
\hline Morus alba & Moraceae & $\mathrm{E}$ & A & 33 & 33 & 0 \\
\hline Nassella filiculmis & Poaceae & End & G & 0 & 0 & 33 \\
\hline Oenothera parodiana & Onagraceae & N & $\mathrm{H}$ & 0 & + & 0 \\
\hline Oplismenus hirtellus ssp. hirtellus & Poaceae & $\mathrm{N}$ & G & 67 & 0 & 0 \\
\hline Opuntia elata & Cactaceae & N & $\mathrm{H}$ & 100 & 0 & + \\
\hline Opuntia salmiana & Cactaceae & $\mathrm{N}$ & $\mathrm{H}$ & 33 & 0 & 0 \\
\hline
\end{tabular}


L. M. Carbone et al. - Cambios florísticos inducidos por el fuego

\begin{tabular}{|c|c|c|c|c|c|c|}
\hline Taxón & Familia & Origen & $\begin{array}{l}\text { Forma de } \\
\text { crecimiento }\end{array}$ & $\begin{array}{c}\text { No } \\
\text { Quemado }\end{array}$ & $\begin{array}{c}\text { Baja } \\
\text { frecuencia } \\
\text { de fuego }\end{array}$ & $\begin{array}{c}\text { Alta } \\
\text { frecuencia } \\
\text { de fuego }\end{array}$ \\
\hline Oxalis conorhyza & Oxalidaceae & $\mathrm{N}$ & $\mathrm{H}$ & 33 & 33 & 0 \\
\hline Oxypetalum arnottianum & Apocynaceae & $\mathrm{N}$ & $\mathrm{H}$ & + & 0 & 0 \\
\hline Oxypetalum coccineum & Apocynaceae & End & $\mathrm{H}$ & 67 & 0 & 0 \\
\hline Oxypetalum crispum & Apocynaceae & $\mathrm{N}$ & $\mathrm{H}$ & + & 0 & 0 \\
\hline Parietaria debilis & Urticaceae & C & $\mathrm{H}$ & + & 0 & 0 \\
\hline Parodia submammulosa & Cactaceae & End & $\mathrm{H}$ & 67 & 67 & 0 \\
\hline Parthenium hysterophorus & Asteraceae & $\mathrm{N}$ & $\mathrm{H}$ & 33 & 0 & 67 \\
\hline Paspalum dilatatum ssp. dilatatum & Poaceae & $\mathrm{N}$ & G & 33 & 0 & 0 \\
\hline Paspalum humboldtianum & Poaceae & $\mathrm{N}$ & G & 0 & 67 & 0 \\
\hline Paspalum malacophyllum & Poaceae & $\mathrm{N}$ & G & 67 & 100 & 100 \\
\hline Paspalum notatum var. notatum & Poaceae & $\mathrm{N}$ & G & 67 & 0 & 33 \\
\hline Paspalum plicatulum & Poaceae & $\mathrm{N}$ & G & 0 & 0 & 33 \\
\hline Passiflora caerulea & Passifloraceae & $\mathrm{N}$ & E & + & 0 & 67 \\
\hline Passiflora morifolia & Passifloraceae & $\mathrm{N}$ & E & 33 & 0 & 0 \\
\hline Pavonia argentina & Malvaceae & $\mathrm{N}$ & A & + & 0 & 0 \\
\hline Pavonia aurigloba & Malvaceae & End & A & 67 & 100 & 100 \\
\hline Pfaffia gnaphaloides & Amaranthaceae & $\mathrm{N}$ & $\mathrm{H}$ & 67 & 67 & 33 \\
\hline Phacelia artemisioides & Boraginaceae & $\mathrm{N}$ & $\mathrm{H}$ & 0 & 0 & + \\
\hline Philibertia gilliesii & Apocynaceae & $\mathrm{N}$ & E & + & 0 & 0 \\
\hline Philibertia tomentosa & Apocynaceae & $\mathrm{N}$ & E & 0 & + & 0 \\
\hline Phyllanthus niruri & Phyllanthaceae & $\mathrm{N}$ & $\mathrm{H}$ & 33 & 0 & 0 \\
\hline Physalis viscosa & Solanaceae & $\mathrm{N}$ & $\mathrm{H}$ & 0 & 0 & + \\
\hline Pinus elliottii & Pinaceae & E & A & 0 & 33 & 0 \\
\hline Piptochaetium montevidense & Poaceae & $\mathrm{N}$ & G & 67 & + & + \\
\hline Plantago tomentosa & Plantaginaceae & N & $\mathrm{H}$ & 0 & + & + \\
\hline Pleopeltis tweediana* & Polypodiaceae & N & $\mathrm{H}$ & + & 0 & 0 \\
\hline Plumbago caerulea & Plumbaginaceae & N & a & 67 & 0 & 0 \\
\hline Podocoma hirsuta & Asteraceae & $\mathrm{N}$ & $\mathrm{H}$ & + & 67 & + \\
\hline Porlieria microphylla & Zygophyllaceae & N & a & 67 & 0 & 0 \\
\hline Porophyllum ruderale & Asteraceae & N & $\mathrm{H}$ & 33 & 0 & 0 \\
\hline Portulaca grandiflora & Portulacaceae & End & $\mathrm{H}$ & 33 & + & 0 \\
\hline Portulaca oleraceae & Portulacaceae & $E$ & $\mathrm{H}$ & + & 0 & 0 \\
\hline Pseudabutilon virgatum & Malvaceae & $\mathrm{N}$ & a & 33 & 0 & 0 \\
\hline Pterocaulon alopecuroides & Asteraceae & $\mathrm{N}$ & $\mathrm{H}$ & 33 & 67 & 33 \\
\hline Puya sphatacea & Bromeliaceae & N & $\mathrm{H}$ & 0 & + & 0 \\
\hline Pyracantha angustifolia & Rosaceae & $E$ & a & + & 0 & 0 \\
\hline Pyracantha atalantioides & Rosaceae & E & a & 33 & + & 0 \\
\hline
\end{tabular}


Bol. Soc. Argent. Bot. 52 (4) 2017

\begin{tabular}{|c|c|c|c|c|c|c|}
\hline Taxón & Familia & Origen & $\begin{array}{l}\text { Forma de } \\
\text { crecimiento }\end{array}$ & $\begin{array}{c}\text { No } \\
\text { Quemado }\end{array}$ & $\begin{array}{c}\text { Baja } \\
\text { frecuencia } \\
\text { de fuego }\end{array}$ & $\begin{array}{c}\text { Alta } \\
\text { frecuencia } \\
\text { de fuego }\end{array}$ \\
\hline Rhynchosia bicentrica & Fabaceae & N & $\mathrm{H}$ & 0 & 67 & 0 \\
\hline Rhynchosia edulis & Fabaceae & N & $E$ & 100 & 100 & 100 \\
\hline Rhynchosia senna var. senna & Fabaceae & End & E & 0 & + & 0 \\
\hline Richardia brasiliensis & Rubiaceae & N & $\mathrm{H}$ & + & + & 0 \\
\hline Rivina humilis & Phytolaccaceae & N & $\mathrm{H}$ & 67 & 0 & 0 \\
\hline Ruprechtia apetala & Polygonaceae & $\mathrm{N}$ & A & 67 & 0 & 0 \\
\hline Salpichroa origanifolia & Solanaceae & $\mathrm{N}$ & $\mathrm{H}$ & 100 & 33 & 0 \\
\hline Schinus fasciculatus & Anacardiaceae & $\mathrm{N}$ & a & 100 & 67 & 33 \\
\hline Schinus longifolius var. Iongifolius & Anacardiaceae & End & a & + & 0 & 0 \\
\hline Schizachyrium microstachyum & Poaceae & $\mathrm{N}$ & G & 0 & 100 & 33 \\
\hline $\begin{array}{l}\text { Schizachyrium salzmannii } \\
\text { var. aristatum }\end{array}$ & Poaceae & N & G & 0 & 67 & 0 \\
\hline Schizachyrium sp. & Poaceae & $\mathrm{N}$ & G & 33 & 0 & 0 \\
\hline Schkuhria pinnata & Asteraceae & $\mathrm{N}$ & $\mathrm{H}$ & 33 & 67 & 67 \\
\hline Sebastiania commersoniana & Euphorbiaceae & $\mathrm{N}$ & A & 67 & 0 & 0 \\
\hline Selaginella sp. & Selaginellaceae & $\mathrm{N}$ & $\mathrm{H}$ & 67 & 33 & 0 \\
\hline Senecio pampeanus & Asteraceae & $\mathrm{N}$ & $\mathrm{H}$ & + & 0 & 0 \\
\hline Senecio vira-vira & Asteraceae & $\mathrm{N}$ & $\mathrm{H}$ & 0 & 0 & 33 \\
\hline Setaria lachnea & Poaceae & $\mathrm{N}$ & G & 33 & 33 & 33 \\
\hline Setaria oblongata & Poaceae & N & G & + & + & 0 \\
\hline Setaria pampeana & Poaceae & $\mathrm{N}$ & G & 67 & 0 & 33 \\
\hline Setaria parviflora var. parviflora & Poaceae & $\mathrm{N}$ & G & 100 & 100 & 67 \\
\hline Sida dyctiocarpa & Malvaceae & $\mathrm{N}$ & $\mathrm{H}$ & 67 & 0 & 67 \\
\hline Sida rhombifolia & Malvaceae & $\mathrm{N}$ & $\mathrm{H}$ & 67 & 100 & 100 \\
\hline Sida spinosa & Malvaceae & $\mathrm{N}$ & $\mathrm{H}$ & 33 & 0 & 33 \\
\hline Sida variegata & Malvaceae & $\mathrm{N}$ & $\mathrm{H}$ & + & 0 & 0 \\
\hline Silene antirrhina var. antirrhina & Caryophyllaceae & $\mathrm{E}$ & $\mathrm{H}$ & 0 & 67 & 67 \\
\hline Simsia dombeyana & Asteraceae & N & $\mathrm{H}$ & + & 0 & 0 \\
\hline Solanum chenopodioides & Solanaceae & End & a & 67 & 0 & 67 \\
\hline Solanum juvenale & Solanaceae & End & $\mathrm{H}$ & 0 & 0 & 33 \\
\hline Solanum palinacanthum & Solanaceae & $\mathrm{N}$ & a & 100 & 33 & 0 \\
\hline Solanum pseudocapsicum & Solanaceae & $\mathrm{N}$ & $\mathrm{H}$ & 33 & 0 & 0 \\
\hline Solanum sisymbriifolium & Solanaceae & $\mathrm{N}$ & $\mathrm{H}$ & 0 & 33 & 0 \\
\hline Solanum stuckertii & Solanaceae & $\mathrm{N}$ & a & + & 0 & 0 \\
\hline Solanum tucumanense & Solanaceae & $\mathrm{N}$ & a & 33 & 0 & 0 \\
\hline Solanum viarum* & Solanaceae & $\mathrm{N}$ & a & 33 & 0 & 0 \\
\hline Solidago chilensis & Asteraceae & $\mathrm{N}$ & $\mathrm{H}$ & 0 & + & 33 \\
\hline Sphaeralcea cordobensis & Malvaceae & End & $\mathrm{H}$ & 0 & 0 & 33 \\
\hline
\end{tabular}


L. M. Carbone et al. - Cambios florísticos inducidos por el fuego

\begin{tabular}{|c|c|c|c|c|c|c|}
\hline Taxón & Familia & Origen & $\begin{array}{l}\text { Forma de } \\
\text { crecimiento }\end{array}$ & $\begin{array}{c}\text { No } \\
\text { Quemado }\end{array}$ & $\begin{array}{c}\text { Baja } \\
\text { frecuencia } \\
\text { de fuego }\end{array}$ & $\begin{array}{c}\text { Alta } \\
\text { frecuencia } \\
\text { de fuego }\end{array}$ \\
\hline Stenandrium diphyllum & Acanthaceae & $\mathrm{N}$ & $\mathrm{H}$ & 0 & 67 & 0 \\
\hline Stevia satureiifolia var. satureiifolia & Asteraceae & End & $\mathrm{H}$ & 0 & 67 & 0 \\
\hline $\begin{array}{l}\text { Stylosanthes montevidensis } \\
\text { var. montevidensis }\end{array}$ & Fabaceae & $\mathrm{N}$ & $\mathrm{H}$ & 33 & 100 & 67 \\
\hline Tagetes minuta & Asteraceae & $\mathrm{N}$ & $\mathrm{H}$ & 33 & 0 & 33 \\
\hline Talinum paniculatum & Talinaceae & E & $\mathrm{H}$ & 33 & 0 & 33 \\
\hline Tillandsia capillaris & Bromeliaceae & N & $\mathrm{H}$ & 100 & 33 & 0 \\
\hline Tragia geraniifolia & Euphorbiaceae & $\mathrm{N}$ & $\mathrm{H}$ & 67 & 100 & 100 \\
\hline Trixis divaricata ssp. discolor & Asteraceae & End & a & + & 0 & 33 \\
\hline Turnera sidoides spp. pinnatifida & Turneraceae & $\mathrm{N}$ & $\mathrm{H}$ & 0 & 67 & 0 \\
\hline Ulmus pumila & Ulmaceae & E & A & 0 & 33 & 0 \\
\hline Verbascum virgatum & Scrophulariaceae & E & $\mathrm{H}$ & 0 & + & + \\
\hline Verbena intermedia & Verbenaceae & N & $\mathrm{H}$ & 0 & + & 0 \\
\hline $\begin{array}{l}\text { Vernonanthura nudiflora } \\
\text { f. nudiflora }\end{array}$ & Asteraceae & $\mathrm{N}$ & $\mathrm{H}$ & 33 & 67 & 100 \\
\hline Vicia setifolia var. setifolia & Fabaceae & N & E & 0 & + & + \\
\hline Vigna caracalla & Fabaceae & $\mathrm{N}$ & E & 33 & 0 & 0 \\
\hline Wahlenbergia linarioides & Campanulaceae & $\mathrm{N}$ & $\mathrm{H}$ & 0 & + & 0 \\
\hline Wedelia buphtalmiflora & Asteraceae & End & $\mathrm{H}$ & 0 & 33 & 33 \\
\hline Wissadula gymnanthemum & Malvaceae & $\mathrm{N}$ & $\mathrm{H}$ & + & 0 & 0 \\
\hline Zanthoxylum coco & Rutaceae & N & A & 33 & 0 & 33 \\
\hline Zinnia peruviana & Asteraceae & $\mathrm{N}$ & $\mathrm{H}$ & 100 & 33 & 67 \\
\hline Zornia latifolia & Fabaceae & $\mathrm{N}$ & $\mathrm{H}$ & 33 & 67 & 0 \\
\hline
\end{tabular}


Bol. Soc. Argent. Bot. 52 (4) 2017

Apéndice 2. Riqueza de especies observada y estimada por índices no paramétricos (media y desvío estándar) para datos de ocurrencia de especies vegetales en cada escenario de fuego. Los estimadores utilizados fueron ICE, basado en la incidencia de especies raras, y Chao2, Jackknife 1 y Jackknife 2, que consideran el número de especies que estuvieron presentes una vez (singletons) o dos veces

(doubletons) en el muestreo.

\begin{tabular}{|cccc|}
\hline $\begin{array}{c}\text { Estimadores no } \\
\text { paramétricos }\end{array}$ & No quemado & Baja frecuencia de fuego & Alta frecuencia de fuego \\
\hline Riqueza observada & 107 & 88 & 64 \\
ICE & $276.2 \pm 0.0$ & $173.3 \pm 0.0$ & $214.2 \pm 0.0$ \\
Chao 2 & $207.7 \pm 17.1$ & $140.3 \pm 8.3$ & $158.1 \pm 17.5$ \\
Jackknife 1 & $206.3 \pm 7.9$ & $153.0 \pm 5.8$ & $150.0 \pm 7.6$ \\
Jackknife 2 & $227.5 \pm 0.0$ & $163.5 \pm 0.0$ & $166.8 \pm 0.0$ \\
\hline
\end{tabular}

